\title{
Singular Control of the Drift of a Brownian System
}

\author{
Salvatore Federico ${ }^{1}(1) \cdot$ Giorgio Ferrari $^{2} \cdot$ Patrick Schuhmann $^{3}$
}

Accepted: 15 April 2021 / Published online: 29 April 2021

(C) The Author(s) 2021

\begin{abstract}
We consider a standard Brownian motion whose drift can be increased or decreased in a possibly singular manner. The objective is to minimize an expected functional involving the time-integral of a running cost and the proportional costs of adjusting the drift. The resulting two-dimensional degenerate singular stochastic control problem has interconnected dynamics and it is solved by combining techniques of viscosity theory and free boundary problems. We provide a detailed description of the problem's value function and of the geometry of the state space, which is split into three regions by two monotone curves. Our main result shows that those curves are continuously differentiable with locally Lipschitz derivative and solve a system of nonlinear ordinary differential equations.
\end{abstract}

Keywords Singular stochastic control · Dynkin game - Viscosity solution · Free boundary $\cdot$ Smooth-fit $\cdot$ Brownian motion · Ordinary differential equation

Mathematics Subject Classification 93E20 · 91A55 - 49L25 · 49J40 · 35R35 - 91B64

$凶 \quad$ Salvatore Federico

salvatore.federico@unige.it

Giorgio Ferrari

giorgio.ferrari@uni-bielefeld.de

Patrick Schuhmann

patrick.schuhmann@uni-bielefeld.de

1 Dipartimento di Economia, Università di Genova, Via Vivaldi 5, 16126 Genova, Italy

2 Center for Mathematical Economics (IMW), Bielefeld University, Universitätsstrasse 25, 33615 Bielefeld, Germany

3 Center for Mathematical Economics (IMW), Bielefeld University, Universitätsstrasse 25, 33615 Bielefeld, Germany 


\section{Introduction}

Consider a system whose position or level is subject to random fluctuations and can be corrected by acting on its drift. The latter can be increased or decreased, and the (cumulative) actions affecting the drift's dynamics are not necessarily absolutely continuous with respect to the Lebesgue measure, as functions of time; also impulses or singularly continuous forces can be applied. The objective of the decision maker is to minimize a total expected functional consisting of the time-integral of a running cost and of the proportional costs of adjusting the drift.

We model this problem as a two-dimensional singular stochastic control problem (see, e.g., [25,29,30], and [45] as classical contributions to the theory of singular stochastic control). The system's position/level $X$ evolves as

$$
X_{t}=x+\alpha \int_{0}^{t} Y_{s} \mathrm{~d} s+\eta W_{t}, \quad x \in \mathbb{R}
$$

for some positive constants $\alpha, \eta$ and for a given standard Brownian motion $W$, and the drift $Y$ is such that

$$
Y_{t}=y+\xi_{t}^{+}-\xi_{t}^{-}, \quad y \in \mathbb{R}
$$

Here, $\xi_{t}^{+}$(respectively, $\xi_{t}^{-}$) are the cumulative increase (respectively, decrease) of the drift up to time $t \geq 0$ and, as such, $\xi^{+}$and $\xi^{-}$are nondecreasing processes, and $\xi:=\xi^{+}-\xi^{-}$has finite variation. The process $X$ might be thought of as a random demand/level of sales whose instantaneous trend $Y$ can be affected via production, according to supply and demand rules, or through an inventory management policy (see, e.g., the review [44]). Alternatively, $X$ could be the position of a satellite which is subject to random disturbances and can be adjusted by properly acting on its velocity. The decision maker aims at picking a control rule $\xi$ that minimizes an expected cost functional. This consists of a term measuring the total cost of acting on the system, which is proportional to the total variation of $\xi$, and of a term involving a running convex cost function $f$ of the current values $\left(X_{t}, Y_{t}\right)$. For example, if $X$ is a satellite position and $Y$ its velocity, the decision maker might want to keep the satellite as close as possible to a given target level, say 0 , while minimizing the system's kinetic energy; in such a case a possible choice of $f$ might therefore be $f(x, y)=x^{2}+y^{2}$. The resulting optimization problem then reads as

$$
\inf _{\xi} \mathrm{E}\left[\int_{0}^{\infty} e^{-\rho t} f\left(X_{t}, Y_{t}\right) \mathrm{d} t+\int_{0}^{\infty} e^{-\rho t} K \mathrm{~d}\left(\xi_{t}^{+}+\xi_{t}^{-}\right)\right],
$$

and it thus takes the form of a two-dimensional degenerate singular stochastic control problem with interconnected dynamics and controls of bounded-variation.

Our problem might be seen as a generalization of the bounded-velocity control of a scalar Brownian motion introduced by Beneš in 1974 [3], which has stimulated a subsequent large literature allowing for different specifications of the performance criterion and incorporating also other features like discretionary stopping and partial 
observation (see [1,4,26-28,34], among many others). However, while in the previous papers the decision maker tracks the position of the Brownian system by choosing the value of its drift within a bounded set (hence the term bounded-velocity control), in our problem the Brownian motion is only indirectly affected by the controller's actions that, in fact, can unlimitedly increase and decrease the Brownian's drift at proportional costs. As a result, in our case the optimal control rule is expected to be of singular type (see Sect. 6.1 below), rather than of so-called bang-bang type (cf. [3,26-28], among others).

In [43] the optimal correction problem of a damped random oscillator is studied. Differently to us, in that paper the velocity is subject to random disturbances and it is linearly controlled via a process of bounded variation, while the oscillator's position is not affected by noise. The authors formulate the problem as a cheap degenerate two-dimensional singular stochastic control problem (i.e. as a problem where the performance criterion does not include the total cost of actions), and a thorough study of the related dynamic programming equation is performed via analytic methods. In [13], it is provided a numerical analysis of the non-cheap linear version of the control problem of [43].

The two papers that are perhaps closest to ours are [32] and [21]. In [32] a singular stochastic control problem with monotone controls and with finite-fuel constraint is considered. The problem is motivated by the issue of irreversible installation of solar panels, where the price of solar electricity is mean-reverting, with drift affected by the cumulative amount of installed solar panels. The authors solve the problem via a guess-and-verify approach and characterize the free boundary as the unique solution to a first-order ordinary differential equation (ODE) complemented by a boundary condition directly implied by the finite-fuel constraint. In [21] a two-dimensional singular stochastic control problem with controls of bounded-variation and interconnected dynamics is studied. The problem's characteristic is that the mean-reversion level of the diffusive component of the state process is an affine function of the purely controlled second component.

Clearly, there is also a large literature on two-dimensional degenerate boundedvariation stochastic control problems where the two components of the state-process are decoupled (see [2,16,20,23,24], and [35], among many others). In those works, it is usually shown that the value function solves the associated dynamic programming principle in the classical sense and the free boundaries are characterized in terms of algebraic/integral equations. These results are obtained through various methods ranging from a "guess-and-verify" approach (see, e.g., [2,16], and [35]), viscosity theory ( [20]), the connection to optimal stopping ([31]), and the link to switching controls ([23] and [24]). However, in our problem, the two dynamics of the state process are interconnected (cf. (1.1) and (1.2)), and this makes the approaches developed in [31] and $[23,24]$ for the connection to optimal stopping games and optimal switching, respectively, not directly applicable. Moreover, given the complexity of the equations arising in our analysis (see Theorem 6.1 below), the verification of the actual optimality of a smooth solution to the dynamic programming equation is also particularly challenging, thus refraining us from the application of a "guess-and-verify" approach.

For the previous reasons, as in [21], we follow here a direct approach which, by employing techniques from viscosity theory and free-boundary problems, enables us 
to provide a detailed study of the value function and of the geometry of the problem's state space. In particular, we show that: (i) the value function $V$ is differentiable with first derivatives that are (locally) Lipschitz, and its $y$-derivative identifies with the value of an optimal stopping game (see also [31] in the case of decoupled dynamics, and [11] for a coupled non degenerate setting); (ii) the two-dimensional state space is split into three connected regions (continuation and action regions) by two monotone curves (free boundaries); (iii) the expression of the value function in each of those regions is provided; (iii) the second order derivative $V_{y x}$ is continuous in the whole space (second-order smooth-fit); (iv) the free boundaries solve a system of integral equations.

Furthermore, because the uncontrolled process is a Brownian motion (rather than a more complex Ornstein-Uhlenbeck process as in [21]), in this paper we are able to push the analysis of [21] much further by providing new results (see Sects. 5 and 6 below). In particular, we can show that the free boundaries delineating action and inaction regions are continuously differentiable with locally Lipschitz derivative (see Theorem 6.4). To our knowledge, in the context of a fully degenerate two-dimensional singular stochastic control problem with interconnected dynamics, a similar finding appears here for the first time. The proof of the aforementioned regularity of the free boundaries hinges on a series of intermediate novel results. First of all, we show that the limit of the third derivative $V_{y x x}$ at the free boundaries along any sequence of points belonging to the (interior of the) continuation region exists and is nonzero (cf. Proposition 5.7). This allows us to apply (a suitable version of) the implicit function theorem and to show that the free boundaries are locally Lipschitz functions of the $y$ coordinate (Proposition 5.8). Then, by exploiting such a property and differentiating the integral equations solved by the free boundaries, we can prove that the latter satisfy a system of (explicitly computable) first-order ODEs. The regularity of the forcing term appearing in the ODEs finally implies that the free boundaries are actually continuously differentiable with locally Lipschitz derivative (see Theorem 6.4). Along with that, we determine explicit expressions for the coefficients $A$ and $B$ appearing in the expression of the value function (cf. Theorem 4.5 and Corollary 6.2). As it is discussed in Remark 6.3, this has not been possible in [21].

Unfortunately, providing boundary conditions complementing the system of ODEs for the free boundaries still remains an open problem. Indeed, it seems hard to identify a relevant value of $y$ for which the values of the free boundaries can be determined, as well as some kind of asymptotic growth that might restrict the functional class where to look for uniqueness of the ODEs' system. However, in Sect. 6.1 we propose a conjecture about the derivation of a Cauchy problem involving the first derivatives of the free boundaries with respect to the parameter $\alpha$ (cf. (1.1)), rather than $y$. A discussion on the structure of the optimal control is also presented in Sect. 6.1.

The rest of the paper is organized as follows. Problem formulation and preliminary results are provided in Sect. 2, while preliminary properties of the free boundaries in Sect. 3. Section 4 contains the structure of the value function and the secondorder smooth-fit property, and most of the results of this section follow from them in [21]. Further important properties of the free boundaries - as their (locally) Lipschitz continuity - are proved in Sect. 5, while the system of ODEs for the free boundaries is finally obtained in Sect. 6. 


\subsection{Notation}

In the rest of this paper, we adopt the following notation and functional spaces. We will use $|\cdot|$ for the Euclidean norm on any finite-dimensional space, without indicating the dimension each time for simplicity of exposition.

Given a smooth function $h: \mathbb{R} \rightarrow \mathbb{R}$, we shall write $h^{\prime}, h^{\prime \prime}$, etc. to denote its derivatives. If the function $h$ admits $k$ continuous derivatives, $k \geq 1$, we shall write $h \in C^{k}(\mathbb{R} ; \mathbb{R})$, while $h \in C(\mathbb{R} ; \mathbb{R})$ if such a function is only continuous.

For a smooth function $h: \mathbb{R}^{2} \rightarrow \mathbb{R}$, we denote by $h_{x}, h_{y}, h_{x x}, h_{y y}$, etc. its partial derivatives. Given $k, j \in \mathbb{N}$, we let $C^{k, j}\left(\mathbb{R}^{2} ; \mathbb{R}\right)$ be the class of functions $h: \mathbb{R}^{2} \rightarrow \mathbb{R}$ which are $k$-times continuously differentiable with respect to the first variable and $j$ times continuously differentiable with respect to the second variable. If $k=j$, we shall simply write $C^{k}\left(\mathbb{R}^{2} ; \mathbb{R}\right)$. Moreover, for a domain $\mathcal{O} \subseteq \mathbb{R}^{d}, d \in\{1,2\}$, we shall work with the space $C_{\text {loc }}^{k, \text { Lip }}(\mathcal{O} ; \mathbb{R}), k \geq 1$, which consists of all the functions $h: \mathcal{O} \rightarrow \mathbb{R}$ that are $k$ times continuously differentiable, with locally-Lipschitz $k$ th-derivative(s).

Also, for $p \geq 1$ we shall denote by $L^{p}(\mathcal{O} ; \mathbb{R})\left(\operatorname{resp} . L_{\text {loc }}^{p}(\mathcal{O} ; \mathbb{R})\right)$ the space of realvalued functions $h: \mathcal{O} \rightarrow \mathbb{R}$ such that $|h|^{p}$ is integrable with respect to the Lebesgue measure on $\mathcal{O}$ (resp. locally integrable on $\mathcal{O}$ ). Finally, for $k \geq 1$, we shall make use of the space $W^{k, p}(\mathcal{O} ; \mathbb{R})\left(\right.$ resp. $\left.W_{\text {loc }}^{k, p}(\mathcal{O} ; \mathbb{R})\right)$, which is the space of all the functions $h: \mathcal{O} \rightarrow \mathbb{R}$ that admit $k$ th-order weak derivative $(\mathrm{s})$ in $\left.L^{p}(\mathcal{O} ; \mathbb{R})\left(\operatorname{resp} . L_{\text {loc }}^{p}(\mathcal{O} ; \mathbb{R})\right)\right)$.

\section{Problem Formulation and Preliminary Results}

Let $\left(\Omega, \mathcal{F}, \mathbb{F}:=\left(\mathcal{F}_{t}\right)_{t \geq 0}, \mathrm{P}\right)$ be a complete filtered probability space rich enough to accommodate an $\mathbb{F}$-Brownian motion $W:=\left(W_{t}\right)_{t \geq 0}$. We assume that the filtration $\mathbb{F}$ satisfies the usual conditions.

We introduce the (nonempty) set

$$
\begin{aligned}
\mathcal{A}:= & \left\{\xi: \Omega \times \mathbb{R}_{+} \rightarrow \mathbb{R}:\left(\xi_{t}\right)_{t \geq 0} \text { is } \mathbb{F} \text {-adapted and such that } t \mapsto \xi_{t}\right. \text { is a.s. } \\
& \text { càdlàg and (locally) of finite variation }\},
\end{aligned}
$$

and for any $\xi \in \mathcal{A}$ we denote by $\xi^{+}$and $\xi^{-}$the two nondecreasing $\mathbb{F}$-adapted càdlàg processes providing the minimal decomposition of $\xi$; that is, such that $\xi=\xi^{+}-\xi^{-}$ and the (random) Borel-measures induced on $[0, \infty)$ by $\xi^{+}$and $\xi^{-}$have disjoint supports. In the following, for any $\xi \in \mathcal{A}$, we set $\xi_{0^{-}}^{ \pm}=0$ a.s. and we denote by $|\xi|_{t}:=\xi_{t}^{+}+\xi_{t}^{-}, t \geq 0$, its total variation.

For $\xi \in \mathcal{A},(x, y) \in \mathbb{R}^{2}$, and $\alpha>0$, we then consider the purely controlled dynamics

$$
Y_{t}^{y, \xi}=y+\xi_{t}^{+}-\xi_{t}^{-}, \quad t \geq 0, Y_{0^{-}}^{y, \xi}=y,
$$


as well as the diffusive

$$
\left\{\begin{array}{l}
\mathrm{d} X_{t}^{x, y, \xi}=\alpha Y_{t}^{y, \xi} \mathrm{d} t+\eta \mathrm{d} W_{t}, \quad t>0 \\
X_{0}^{x, y, \xi}=x
\end{array}\right.
$$

The unique strong solution to (2.3) is given by

$$
X_{t}^{x, y, \xi}=x+\alpha \int_{0}^{t} Y_{s}^{y, \xi} \mathrm{d} s+\eta W_{t}, \quad \forall \xi \in \mathcal{A}, t \geq 0 .
$$

The parameter $\alpha$ measures the strength of the interaction between the processes $X$ and $Y$. Clearly, for $\alpha=0$ the two dynamics are decoupled and $X$ is a Brownian motion with volatility $\eta>0$.

Remark 2.1 It is worth noticing that the restriction $\alpha>0$ is not necessary for the subsequent analysis; in fact, all the results of this paper (up to obvious modifications) can be still deduced with the same techniques also in the case $\alpha<0$. We have decided to consider only the case $\alpha>0$ just in order to simplify the exposition.

Controlling the dynamics $(X, Y)$ gives rise to an instantaneous cost that is proportional - with marginal constant cost $K>0$ - to the total variation of the exerted control. Moreover, the controller faces also a running cost depending on the current levels $\left(X_{t}, Y_{t}\right)$. The aim is therefore to choose a control $\xi \in \mathcal{A}$ such that, for any $(x, y) \in \mathbb{R}^{2}$, and for a given $\rho>0$, the cost functional

$$
\mathcal{J}(x, y ; \xi):=\mathrm{E}\left[\int_{0}^{\infty} e^{-\rho t} f\left(X_{t}^{x, y, \xi}, Y_{t}^{y, \xi}\right) \mathrm{d} t+\int_{0}^{\infty} e^{-\rho t} K \mathrm{~d}|\xi|_{t}\right]
$$

is minimized; that is, to solve

$$
V(x, y):=\inf _{\xi \in \mathcal{A}} \mathcal{J}(x, y ; \xi), \quad(x, y) \in \mathbb{R}^{2}
$$

In (2.5) and in the following, the integrals with respect to $\mathrm{d}|\xi|$ and $\mathrm{d} \xi^{ \pm}$are intended in the Lebesgue-Stieltjes' sense; in particular, for $\zeta \in\left\{|\xi|, \xi^{+}, \xi^{-}\right\}$, we set $\int_{0}^{s}(\cdot) \mathrm{d} \zeta_{t}:=\int_{[0, s]}(\cdot) \mathrm{d} \zeta_{t}$ in order to take into account a possible mass at time zero of the Borel (random) measure $\mathrm{d} \zeta$. The function $f: \mathbb{R}^{2} \rightarrow \mathbb{R}^{+}$satisfies the following standing assumption.

Assumption 2.2 There exists constants $p>1$, and $C_{0}, C_{1}, C_{2}>0$ such that the following hold true:

(i) $0 \leq f(z) \leq C_{0}(1+|z|)^{p}$, for every $z=(x, y) \in \mathbb{R}^{2}$;

(ii) for every $z=(x, y), z^{\prime}=\left(x^{\prime}, y^{\prime}\right) \in \mathbb{R}^{2}$,

$$
\left|f(z)-f\left(z^{\prime}\right)\right| \leq C_{1}\left(1+f(z)+f\left(z^{\prime}\right)\right)^{1-\frac{1}{p}}\left|z-z^{\prime}\right|
$$


(iii) for every $z=(x, y), z^{\prime}=\left(x^{\prime}, y^{\prime}\right) \in \mathbb{R}^{2}$ and $\lambda \in(0,1)$,

$$
\begin{aligned}
0 & \leq \lambda f(z)+(1-\lambda) f\left(z^{\prime}\right)-f\left(\lambda z+(1-\lambda) z^{\prime}\right) \\
& \leq C_{2} \lambda(1-\lambda)\left(1+f(z)+f\left(z^{\prime}\right)\right)^{\left(1-\frac{2}{p}\right)^{+}}\left|z-z^{\prime}\right|^{2}
\end{aligned}
$$

(iv) $x \mapsto f_{y}(x, y)$ is nondecreasing for any $y \in \mathbb{R}$.

Remark 2.3 (i) By Assumption 2.2-(iii), $f$ is convex and locally semiconcave; then, by [9, Cor. 3.3.8],

$$
f \in C_{\mathrm{loc}}^{1, \text { Lip }}\left(\mathbb{R}^{2} ; \mathbb{R}\right)=W_{\text {loc }}^{2, \infty}\left(\mathbb{R}^{2} ; \mathbb{R}\right)
$$

(ii) A function $f$ satisfying Assumption 2.2 is, for example,

$$
f(x, y)=|x-\hat{x}|^{p}+|y-\hat{y}|^{p},
$$

with $p \geq 2$ ad for some $\hat{x}, \hat{y} \in \mathbb{R}$.

We now provide some preliminary properties of the value function, whose classical proof exploits the linear structure of the state equations.

Proposition 2.4 Let Assumption 2.2 hold and let $p>1$ be the constant appearing in such assumption. There exist constants $\widehat{C}_{0}, \widehat{C}_{1}, \widehat{C}_{2}>0$ such that the following hold:

(i) $0 \leq V(z) \leq \widehat{C}_{0}\left(1+|z|^{p}\right)$, for every $z=(x, y) \in \mathbb{R}^{2}$;

(ii) for every $z=(x, y), z^{\prime}=\left(x^{\prime}, y^{\prime}\right) \in \mathbb{R}^{2}$,

$$
\left|V(z)-V\left(z^{\prime}\right)\right| \leq \widehat{C}_{1}\left(1+|z|+\left|z^{\prime}\right|\right)^{p-1}\left|z-z^{\prime}\right|
$$

(iii) for every $z=(x, y), z^{\prime}=\left(x^{\prime}, y^{\prime}\right) \in \mathbb{R}^{2}$ and $\lambda \in(0,1)$,

$$
\begin{aligned}
0 & \leq \lambda V(z)+(1-\lambda) V\left(z^{\prime}\right)-V\left(\lambda z+(1-\lambda) z^{\prime}\right) \\
& \leq \widehat{C}_{2} \lambda(1-\lambda)\left(1+|z|+\left|z^{\prime}\right|\right)^{(p-2)^{+}}\left|z-z^{\prime}\right|^{2}
\end{aligned}
$$

In particular, by (iii), $V$ is convex and locally semiconcave, hence, by Corollary 3.3.8 in [9],

$$
V \in C_{l o c}^{1, \text { Lip }}\left(\mathbb{R}^{2} ; \mathbb{R}\right)=W_{\text {loc }}^{2, \infty}\left(\mathbb{R}^{2} ; \mathbb{R}\right)
$$

Proof Due to (2.2) and (2.3), the properties of $f$ required in (ii) and (iii) of Assumption 2.2 are straightly inherited by $V$ (see, e.g., the proof of Theorem 1 of [12], that can easily adapted to our infinite time-horizon setting, or that of Theorem 2.1 in [10]). 


\section{The Dynkin Game and Preliminary Properties of the Free Boundaries}

In this section we show that $V_{y}$ identifies with the value function of a suitable Dynkin game (a zero-sum game of optimal stopping), and we derive preliminary properties of the two curves (free boundaries) that delineate the region of the space where the $\left|V_{y}\right|<K$. In order to simplify the notation, in the following we write $X^{x, y}$, instead of $X^{x, y, 0}$, to identify the solution to (2.3) for $\xi \equiv 0$. Most of the results of this section are close to those in Section 3 of [21], and their proof will be therefore omitted for the sake of brevity.

Theorem 3.1 Let $(x, y) \in \mathbb{R}^{2}$. Denote by $\mathcal{T}$ the set of all $\mathbb{F}$-stopping times, and for $(\sigma, \tau) \in \mathcal{T} \times \mathcal{T}$ consider the stopping functional

$$
\begin{aligned}
\Psi(\sigma, \tau ; x, y):= & E\left[\int_{0}^{\tau \wedge \sigma} e^{-\rho t}\left(f_{y}\left(X_{t}^{x, y}, y\right)+\alpha V_{x}\left(X_{t}^{x, y}, y\right)\right) \mathrm{d} t\right. \\
& \left.-e^{-\rho \tau} K \mathbb{1}_{\{\tau<\sigma\}}+e^{-\rho \sigma} K \mathbb{1}_{\{\tau>\sigma\}}\right],
\end{aligned}
$$

where $V_{x}$ is the partial derivative of $V$ with respect to $x$ (which exists and is continuous by Proposition 2.4). One has that the game has a value, i.e.

$$
\inf _{\sigma \in \mathcal{T}} \sup _{\tau \in \mathcal{T}} \Psi(\sigma, \tau ; x, y)=\sup _{\tau \in \mathcal{T}} \inf _{\sigma \in \mathcal{T}} \Psi(\sigma, \tau ; x, y),
$$

and such a value is given by

$$
V_{y}(x, y)=\inf _{\sigma \in \mathcal{T}} \sup _{\tau \in \mathcal{T}} \Psi(\sigma, \tau ; x, y)=\sup _{\tau \in \mathcal{T}} \inf _{\sigma \in \mathcal{T}} \Psi(\sigma, \tau ; x, y)
$$

Moreover, the couple of $\mathbb{F}$-stopping times $\left(\tau^{\star}(x, y), \sigma^{\star}(x, y)\right):=\left(\tau^{\star}, \sigma^{\star}\right)$ such that

$$
\sigma^{\star}:=\inf \left\{t \geq 0: V_{y}\left(X_{t}^{x, y}, y\right) \geq K\right\}, \quad \tau^{\star}:=\inf \left\{t \geq 0: V_{y}\left(X_{t}^{x, y}, y\right) \leq-K\right\}
$$

(with the usual convention $\inf \emptyset=+\infty$ ) form a saddle-point; that is,

$\forall \tau \in \mathcal{T} \quad \Psi\left(\sigma^{\star}, \tau ; x, y\right) \leq V_{y}(x, y)=\Psi\left(\sigma^{\star}, \tau^{\star} ; x, y\right) \leq \Psi\left(\sigma, \tau^{\star} ; x, y\right) \quad \forall \sigma \in \mathcal{T}$.

Notice that the connection between bounded-variation control problems and Dynkin games is established in great generality when either the components of the state process are decoupled (see [31] and [23,24]), or when the controlled system is one-dimensional (see [5]). In our two-dimensional setting with interconnected dynamics, the approaches followed in the aforementioned works is not applicable. We instead prove Theorem 3.1 by employing Theorems 3.11 and 3.13 in [11], through a suitable (and not immediate) approximation procedure needed to accommodate our degenerate setting. Since the 
proof of Theorem 3.1 is completely analogous to that developed in order to prove Theorem 3.1 in [21] (see Appendix A therein), we omit the details.

From (3.2) it readily follows that $-K \leq V_{y}(x, y) \leq K$ for any $(x, y) \in \mathbb{R}^{2}$. Hence, defining

$$
\begin{cases}\mathcal{I}:=\left\{(x, y) \in \mathbb{R}^{2}:\right. & \left.V_{y}(x, y)=-K\right\}, \\ \mathcal{C}:=\left\{(x, y) \in \mathbb{R}^{2}:\right. & \left.-K<V_{y}(x, y)<K\right\}, \\ \mathcal{D}:=\left\{(x, y) \in \mathbb{R}^{2}:\right. & \left.V_{y}(x, y)=K\right\},\end{cases}
$$

we have that those regions provide a partition of $\mathbb{R}^{2}$.

By continuity of $V_{y}$ (cf. Proposition 2.4), $\mathcal{C}$ is an open set, while $\mathcal{I}$ and $\mathcal{D}$ are closed sets. Moreover, convexity of $V$ provides the representation

$$
\begin{array}{r}
\mathcal{C}=\left\{(x, y) \in \mathbb{R}^{2}: b_{1}(x)<y<b_{2}(x)\right\}, \\
\mathcal{I}=\left\{(x, y) \in \mathbb{R}^{2}: y \leq b_{1}(x)\right\}, \quad \mathcal{D}=\left\{(x, y) \in \mathbb{R}^{2}: y \geq b_{2}(x)\right\},
\end{array}
$$

where the functions $b_{1}: \mathbb{R} \rightarrow \overline{\mathbb{R}}$ and $b_{2}: \mathbb{R} \rightarrow \overline{\mathbb{R}}$ are defined as

$$
\begin{aligned}
& b_{1}(x):=\inf \left\{y \in \mathbb{R} \mid V_{y}(x, y)>-K\right\}=\sup \left\{y \in \mathbb{R} \mid V_{y}(x, y)=-K\right\}, \quad x \in \mathbb{R}, \\
& b_{2}(x):=\sup \left\{y \in \mathbb{R} \mid V_{y}(x, y)<K\right\}=\inf \left\{y \in \mathbb{R} \mid V_{y}(x, y)=K\right\}, \quad x \in \mathbb{R}, \text { (3.6) }
\end{aligned}
$$

(with the usual conventions $\inf \emptyset=\infty, \inf \mathbb{R}=-\infty, \sup \emptyset=-\infty, \sup \mathbb{R}=\infty$ ).

Equation (3.2), together with the fact that $x \mapsto V_{x}(x, y)$ is nondecreasing for any $y \in \mathbb{R}$ by convexity of $V$ (cf. Proposition 2.4) and $x \mapsto f_{y}(x, y)$ is nondecreasing by Assumption 2.2-(iv), easily imply the following result.

Lemma 3.2 $V_{y}(\cdot, y)$ is nondecreasing for all $y \in \mathbb{R}$.

We now move on by obtaining preliminary properties of $b_{1}$ and $b_{2}$. Its proof can be obtained by exploiting the continuity and the monotonicity of $V_{y}$, and easily adjusting the arguments of the proof of Proposition 3.3 of [21] to the present setting in which $V_{y}(\cdot, y)$ is nondecreasing.

Proposition 3.3 The following hold:

(i) $b_{1}: \mathbb{R} \rightarrow \mathbb{R} \cup\{-\infty\}, b_{2}: \mathbb{R} \rightarrow \mathbb{R} \cup\{\infty\}$;

(ii) $b_{1}$ and $b_{2}$ are nonincreasing;

(iii) $b_{1}(x)<b_{2}(x)$ for all $x \in \mathbb{R}$;

(iv) $b_{1}$ is left-continuous and $b_{2}$ is right-continuous.

Let us now define

$$
\bar{b}_{1}:=\sup _{x \in \mathbb{R}} b_{1}(x), \quad \underline{b}_{1}:=\inf _{x \in \mathbb{R}} b_{1}(x), \quad \bar{b}_{2}:=\sup _{x \in \mathbb{R}} b_{2}(x), \quad \underline{b}_{2}:=\inf _{x \in \mathbb{R}} b_{2}(x),
$$


together with the pseudo-inverses of $b_{1}$ and $b_{2}$ by

$$
g_{1}(y):=\sup \left\{x \in \mathbb{R}: b_{1}(x) \geq y\right\}, \quad g_{2}(y):=\inf \left\{x \in \mathbb{R}: b_{2}(x) \leq y\right\}
$$

(again, with the usual conventions $\inf \emptyset=\infty, \inf \mathbb{R}=-\infty, \sup \emptyset=-\infty, \sup \mathbb{R}=$ $\infty)$.

Also the next proposition can be proved by easily adapting to our setting the proof of Proposition 3.4 in [21].

Proposition 3.4 The following holds:

(i) $g_{1}(y)=\inf \left\{x \in \mathbb{R}: V_{y}(x, y)>-K\right\}, \quad g_{2}(y)=\sup \left\{x \in \mathbb{R}: V_{y}(x, y)<K\right\}$;

(ii) the functions $g_{1}, g_{2}$ are nonincreasing and $g_{1}(y)<g_{2}(y)$ for any $y \in \mathbb{R}$;

(iii) If $\bar{b}_{2}<\infty$, then $g_{2}(y)=-\infty$ for all $y \geq \bar{b}_{2}$ and if $\underline{b}_{1}>-\infty$, then $g_{1}(r)=\infty$ for all $y \leq \underline{b}_{1}$.

\section{The Structure of the Value Function and the Second-Order Smooth} Fit

In this section, we exploit the results of the previous section in order to determine the structure of the value function $V$, and to show that $V_{y x}$ is continuous on the whole state space (second-order smooth-fit property).

For any given and fixed $y \in \mathbb{R}$, denote by $\mathcal{L}^{y}$ the infinitesimal generator associated to the uncontrolled process $X^{x, y}$. Acting on $g \in C^{2}(\mathbb{R} ; \mathbb{R})$ it yields

$$
\left(\mathcal{L}^{y} g\right)(x):=\frac{\eta^{2}}{2} g^{\prime \prime}(x)+\alpha y g^{\prime}(x), \quad x \in \mathbb{R} .
$$

Any solution $\beta(\cdot, y)$ to the second-order ordinary differential equation (ODE)

$$
\left(\mathcal{L}^{y} \beta(\cdot, y)\right)(x)-\rho \beta(x, y)=0, \quad x \in \mathbb{R},
$$

can be written as

$$
\beta(x, y)=A(y) \psi(x, \alpha y)+B(y) \varphi(x, \alpha y), \quad x \in \mathbb{R},
$$

where the strictly positive functions $\psi$ and $\varphi$ are given, for any $z \in \mathbb{R}$, by

$$
\psi(x, z):=e^{r_{1}(z) x} \quad \varphi(x, z):=e^{r_{2}(z) x},
$$

with

$$
\begin{aligned}
& r_{1}(z):=\frac{-z+\sqrt{z^{2}+2 \rho \eta^{2}}}{\eta^{2}}>0, \\
& r_{2}(z):=\frac{-z-\sqrt{z^{2}+2 \rho \eta^{2}}}{\eta^{2}}<0 .
\end{aligned}
$$


Notice that $\psi(\cdot, z)$ is strictly increasing while $\varphi(\cdot, z)$ is strictly decreasing for any $z \in \mathbb{R}$.

By the dynamic programming principle, we expect that $V$ identifies with a suitable solution to the following variational inequality

$$
\begin{aligned}
& \max \left\{-v_{y}(x, y)-K, v_{y}(x, y)-K,\left[\left(\rho-\mathcal{L}^{y}\right) v(\cdot, y)\right](x)-f(x, y)\right\}=0 \\
& (x, y) \in \mathbb{R}^{2} .
\end{aligned}
$$

We now show that $V$ is a viscosity solution to (4.4). Later, this will enable us to determine the structure of $V$ (see Proposition 4.5 below) and then to upgrade its regularity (cf. Proposition 4.7) in order to derive necessary optimality conditions for the boundaries splitting the state space (cf. Theorem 6.1).

Definition 4.1 (i) A function $v \in C^{0}\left(\mathbb{R}^{2} ; \mathbb{R}\right)$ is called a viscosity subsolution to (4.4) if, for every $(x, y) \in \mathbb{R}^{2}$ and every $\beta \in C^{2,1}\left(\mathbb{R}^{2} ; \mathbb{R}\right)$ such that $v-\beta$ attains a local maximum at $(x, y)$, it holds

$$
\max \left\{-\beta_{y}(x, y)-K, \beta_{y}(x, y)-K, \rho \beta(x, y)-\left[\mathcal{L}^{y} \beta(\cdot, y)\right](x)-f(x, y)\right\} \leq 0 \text {. }
$$

(ii) A function $v \in C^{0}\left(\mathbb{R}^{2} ; \mathbb{R}\right)$ is called a viscosity supersolution to (4.4) if, for every $(x, y) \in \mathbb{R}^{2}$ and every $\beta \in C^{2,1}\left(\mathbb{R}^{2} ; \mathbb{R}\right)$ such that $v-\beta$ attains a local minimum at $(x, y)$, it holds

$$
\max \left\{-\beta_{y}(x, y)-K, \beta_{y}(x, y)-K, \rho \beta(x, y)-\left[\mathcal{L}^{y} \beta(\cdot, y)\right](x)-f(x, y)\right\} \geq 0 .
$$

(iii) A function $v \in C^{0}\left(\mathbb{R}^{2} ; \mathbb{R}\right)$ is called a viscosity solution to (4.4) if it is both a viscosity subsolution and supersolution.

Following the arguments developed in Theorem 5.1 in Section VIII.5 of [22], one can show the following result (see also Proposition 4.2 in [21])

Proposition 4.2 The value function $V$ is a viscosity solution to (4.4).

Remark 4.3 Recall that by Proposition 2.4-(iii) our value function $V$ lies in the class $W_{\text {loc }}^{2, \infty}\left(\mathbb{R}^{2} ; \mathbb{R}\right)$. Hence, by Lemma 5.4 in Chapter 4 of [46] it is also a strong solution to (4.4) (in the sense, e.g., of [7]; see the same reference also for relations between these notions of solutions); that is, it solves (4.4) in the pointwise sense almost everywhere.

We have decided to employ the concept of viscosity solution since our analysis will later make use of the variational inequality (4.4) on sets of null Lebesgue measure (regular lines) (see Proposition 4.4 and Proposition 4.7 below). Because the viscosity property holds for all (and not merely for a.e.) points of the state space $\mathbb{R}^{2}$, the concept of viscosity solution is still able to provide information on $V$ on regular lines.

For later use, notice that the function

$$
\widehat{V}(x, y):=\mathcal{J}(x, y, 0)=\mathrm{E}\left[\int_{0}^{\infty} e^{-\rho t} f\left(X_{t}^{x, y}, y\right) \mathrm{d} t\right], \quad(x, y) \in \mathbb{R}^{2},
$$


is finite by Assumption 2.2-(i) and standard estimates, and continuously differentiable with respect to $y$ and $x$, given the assumed regularity of $f_{x}$ and $f_{y}$ in Assumption 2.2-(iii). Moreover, for any given and fixed $y \in \mathbb{R}$, we introduce the scale function density of the process $X^{x, y}$

$$
S_{x}(x, z):=\exp \left\{-\frac{2 z x}{\eta^{2}}\right\}, \quad x \in \mathbb{R}
$$

the density of the speed measure

$$
m_{x}(x, z):=\frac{2}{\eta^{2} S_{x}(x, z)}, \quad x \in \mathbb{R}
$$

as well as the positive constant (normalized) Wronskian between $\psi$ and $\varphi$

$$
W:=\frac{\psi_{x}(x, z) \varphi(x, z)-\varphi_{x}(x, z) \psi(x, z)}{S_{x}(x, z)}, \quad(x, z) \in \mathbb{R}^{2} .
$$

Then, letting

$$
G(x, z, u):=W^{-1} \cdot\left\{\begin{array}{l}
\psi(x, u) \varphi(z, u), x \leq z \\
\varphi(x, u) \psi(z, u), x \geq z
\end{array}\right.
$$

be the Green function, we have that $\widehat{V}$ admits the representation (cf., e.g., Ch. 2 of [6])

$$
\widehat{V}(x, y)=\int_{-\infty}^{+\infty} f(z, y) G(x, z, \alpha y) m_{x}(z, \alpha y) \mathrm{d} z, \quad(x, y) \in \mathbb{R}^{2}
$$

that is, using (4.1) and (4.7),

$$
\widehat{V}(x, y)=\frac{2}{\eta^{2} W}\left[e^{r_{1}(\alpha y) x} \int_{x}^{\infty} e^{-r_{1}(\alpha y) z} f(z, y) \mathrm{d} z+e^{r_{2}(\alpha y) x} \int_{-\infty}^{x} e^{-r_{2}(\alpha y) z} f(z, y) \mathrm{d} z\right] .
$$

By direct calculations, it thus follows from (4.11) that $\widehat{V}$ identifies with a classical particular solution to the inhomogeneous linear ODE

$$
\left[\left(\mathcal{L}^{y}-\rho\right) \beta(\cdot, y)\right](x)+f(x, y)=0, \quad x \in \mathbb{R} .
$$

Recall now the regions $\mathcal{C}, \mathcal{I}$ and $\mathcal{D}$ from (3.4), and that $V_{y}=-K$ on $\mathcal{I}$, while $V_{y}=K$ on $\mathcal{D}$. The next proposition provides the structure of $V$ inside $\mathcal{C}$. Its proof can be obtained by arguing exactly as in the proof of Proposition 4.4 of [21] (see also Remarks 4.3 and 4.5 therein), and it is therefore omitted.

Proposition 4.4 Recall (3.7) and let $y_{o} \in\left(\underline{b}_{1}, \bar{b}_{2}\right)$. 
(i) The function $V\left(\cdot, y_{o}\right)$ is a viscosity solution to

$$
\rho \beta\left(x, y_{o}\right)-\left[\mathcal{L}^{y_{o}} \beta\left(\cdot, y_{o}\right)\right](x)-f\left(x, y_{o}\right)=0, \quad x \in\left(g_{1}\left(y_{o}\right), g_{2}\left(y_{o}\right)\right) .
$$

(ii) $V\left(\cdot, y_{o}\right) \in C_{l o c}^{3, L i p}\left(\left(g_{1}\left(y_{o}\right), g_{2}\left(y_{o}\right)\right) ; \mathbb{R}\right)$.

(iii) There exist constants $A\left(y_{o}\right)$ and $B\left(y_{o}\right)$ such that for all $x \in\left(g_{1}\left(y_{o}\right), g_{2}\left(y_{o}\right)\right)$

$$
V\left(x, y_{o}\right)=A\left(y_{o}\right) \psi\left(x, \alpha y_{o}\right)+B\left(y_{o}\right) \varphi\left(x, \alpha y_{o}\right)+\widehat{V}\left(x, y_{o}\right),
$$

where the functions $\psi$ and $\varphi$ have been defined in (4.1) and $\widehat{V}$ is as in (4.5).

We can now determine the structure of the value function $V$. The proof of the next proposition is completely analogous to that of Theorem 4.6 in [21]; however, we provide it here since it will be useful in the proof of a subsequent result (cf. Proposition 5.8).

Proposition 4.5 Define the sets

$$
\mathcal{O}_{1}:=\left\{x \in \mathbb{R}: b_{1}(x)>-\infty\right\} \quad \mathcal{O}_{2}:=\left\{x \in \mathbb{R}: b_{2}(x)<\infty\right\}
$$

There exist functions

$$
A, B \in W_{l o c}^{2, \infty}\left(\left(\underline{b}_{1}, \bar{b}_{2}\right) ; \mathbb{R}\right)=C_{l o c}^{1, L i p}\left(\left(\underline{b}_{1}, \bar{b}_{2}\right) ; \mathbb{R}\right), \quad z_{1,2}: \mathcal{O}_{1,2} \rightarrow \mathbb{R}
$$

such that the value function defined in (2.6) can be written as

$$
V(x, y)= \begin{cases}A(y) \psi(x, \alpha y)+B(y) \varphi(x, \alpha y)+\widehat{V}(x, y) & \text { on } \overline{\mathcal{C}}, \\ z_{1}(x)-K y & \text { on } \mathcal{I}, \\ z_{2}(x)+K y & \text { on } \mathcal{D}\end{cases}
$$

where $\overline{\mathcal{C}}$ denotes the closure of $\mathcal{C}$,

$$
z_{1}(x):=V\left(x, b_{1}(x)\right)+K b_{1}(x), \quad x \in \mathcal{O}_{1}
$$

and

$$
z_{2}(x):=V\left(x, b_{2}(x)\right)-K b_{2}(x), \quad x \in \mathcal{O}_{2} .
$$

Proof We start by deriving the structure of $V$ within $\mathcal{C}$. Using Lemma 4.4, we already know the existence of functions $A, B:\left(\underline{b}_{1}, \bar{b}_{2}\right) \rightarrow \mathbb{R}$ such that

$$
V(x, y)=A(y) \psi(x, \alpha y)+B(y) \varphi(x, \alpha y)+\widehat{V}(x, y), \quad(x, y) \in \mathcal{C} .
$$


Take now $y_{o} \in\left(\underline{b}_{1}, \bar{b}_{2}\right)$. Since $g_{1}(y)<g_{2}(y)$ for any $y \in \mathbb{R}$ (cf. Proposition 3.4(ii)), we can find $x$ and $\tilde{x}, x \neq \tilde{x}$, such that $(x, y),(\tilde{x}, y) \in \mathcal{C}$ for any given $y \in$ $\left(y_{o}-\varepsilon, y_{o}+\varepsilon\right)$, for a suitably small $\varepsilon>0$. Now, by evaluating (4.18) at the points $(x, y)$ and $(\tilde{x}, y)$, we obtain a linear algebraic system that we can solve with respect to $A(y)$ and $B(y)$ so to obtain

$$
\begin{aligned}
& A(y)=\frac{(V(x, y)-\widehat{V}(x, y)) \varphi(\tilde{x}, \alpha y)-(V(\tilde{x}, y)-\widehat{V}(\tilde{x}, y) \varphi(x, \alpha y)}{\psi(x, \alpha y) \varphi(\tilde{x}, \alpha y)-\psi(\tilde{x}, \alpha y) \varphi(x, \alpha y)} \\
& B(y)=\frac{(V(\tilde{x}, y)-\widehat{V}(\tilde{x}, y) \psi(x, \alpha y)-(V(x, y)-\widehat{V}(x, y)) \psi(\tilde{x}, \alpha y)}{\psi(x, \alpha y) \varphi(\tilde{x}, \alpha y)-\psi(\tilde{x}, \alpha y) \varphi(x, \alpha y)}
\end{aligned}
$$

The denominators of the last two expressions do not vanish due to the strict monotonicity of $\psi$ and $\varphi$, and to the fact that $x \neq \tilde{x}$. Since $y_{o}$ was arbitrary and $V, \widehat{V}$, $V_{y}$, and $\widehat{V}_{y}$ are continuous with respect to $y$, we therefore obtain that $A$ and $B$ belong to $W_{\text {loc }}^{2, \infty}\left(\left(\underline{b}_{1}, \bar{b}_{2}\right) ; \mathbb{R}\right)=C_{\text {loc }}^{1, \operatorname{Lip}}\left(\left(\underline{b}_{1}, \bar{b}_{2}\right) ; \mathbb{R}\right)$. The structure of $V$ in the closure of $\mathcal{C}$, denoted by $\overline{\mathcal{C}}$, is then obtained by Proposition 4.4 and by recalling that $V$ is continuous on $\mathbb{R}^{2}$ and that $A, B$, and $\widehat{V}$ are also continuous.

Given the definition of $z_{1}$ and $z_{2}$, the structure of $V$ inside the regions $\mathcal{I}$ and $\mathcal{D}$ follow by (3.4) and the continuity of $V$.

Remark 4.6 Actually, by (4.19) and (4.20) one has that $A$ and $B$ belong to $W^{2, \infty}$ up to $\underline{b}_{1}$ (resp. $\bar{b}_{2}$ ) if $\underline{b}_{1}$ (resp. $\bar{b}_{2}$ ) is finite (cf. also Remark 4.7 in [21]).

Notice that

$$
V_{y x}(x, y)=0 \quad \forall(x, y) \in \mathbb{R}^{2} \backslash \overline{\mathcal{C}} .
$$

The next result shows that one actually has continuity of $V_{y x}$ on the whole $\mathbb{R}^{2}$. Its proof can be obtained by following that of Theorem 5.1 in [21] (see also Proposition 5.3 in [20]), upon recalling that in our setting $V_{y}(\cdot, y)$ is nondecreasing (cf. Lemma 3.2).

Proposition 4.7 One has that

$$
\lim _{\substack{(x, y) \rightarrow\left(x_{o}, y_{o}\right) \\(x, y) \in \mathcal{C}}} V_{y x}(x, y)=0 \quad \forall\left(x_{o}, y_{o}\right) \in \partial \mathcal{C} .
$$

Hence, $V_{y x} \in C\left(\mathbb{R}^{2} ; \mathbb{R}\right)$.

Lemma 4.8 It holds $V_{y x x} \in L_{\text {loc }}^{\infty}\left(\mathbb{R} \times\left(\underline{b}_{1}, \bar{b}_{2}\right) ; \mathbb{R}\right)$.

Proof Notice that by (4.1) one has $\psi_{x x}(x, \alpha y)=r_{1}^{2}(\alpha y) \psi(x, \alpha y), \varphi_{x x}(x, \alpha y)=$ $r_{2}^{2}(\alpha y) \varphi(x, \alpha y)$, and $\psi_{z x x}(x, \alpha y)=r_{1}^{\prime}(\alpha y) r_{1}(\alpha y) \psi(x, \alpha y)\left(2+x r_{1}(\alpha y)\right), \varphi_{z x x}(x, \alpha y)=$ $r_{2}^{\prime}(\alpha y) r_{2}(\alpha y) \varphi(x, \alpha y)\left(2+x r_{2}(\alpha y)\right)$. Moreover, $\widehat{V}_{y x x} \in L_{\mathrm{loc}}^{\infty}\left(\mathbb{R}^{2}\right)$ by direct calculations on $(4.11)$, and $A_{y}, B_{y} \in W_{\mathrm{loc}}^{1, \infty}\left(\left(\underline{b}_{1}, \bar{b}_{2}\right) ; \mathbb{R}\right)$ by Proposition 4.5. Hence, $V_{y x x} \in L_{\mathrm{loc}}^{\infty}\left(\mathbb{R} \times\left(\underline{b}_{1}, \bar{b}_{2}\right) ; \mathbb{R}\right)$ by $(4.15)$. 


\section{Further Properties of the Free Boundaries}

In this section we move on by proving further properties of the free boundaries under additional mild requirements on $f$. The main aim of the following analysis is to determine a local Lipschitz regularity of the free boundaries $g_{1}$ and $g_{2}$ (cf. Proposition 5.8). Exploiting this regularity, in Sect. 6 we will show that $g_{1}$ and $g_{2}$ must solve a system of first-order ordinary differential equations in the classical sense.

Throughout the rest of this paper, the following assumption is in place.

Assumption 5.1 (i) $\lim _{x \rightarrow \pm \infty} f_{x}(x, y)= \pm \infty$.

(ii) $f_{y x}$ exists and it is continuous.

(iii) One of the following holds true:

(a) $x \mapsto f_{y}(x, y)$ is strictly increasing for any $y \in \mathbb{R}$;

(b) $f_{y x} \equiv 0$ and $f(\cdot, y)$ is strictly convex for any $y \in \mathbb{R}$.

Remark 5.2 The functions $f$ discussed in Remark 2.3 satisfy the previous assumptions.

We start by studying the limiting behavior of the functions $b_{i}$ and some natural strict bounds for $g_{i}$.

Proposition 5.3 (i) Let Assumption 5.1-(i) hold. Then

$$
\bar{b}_{1}=\lim _{x \downarrow-\infty} b_{1}(x)=\infty, \quad \underline{b}_{2}=\lim _{x \uparrow \infty} b_{2}(x)=-\infty
$$

hence, by Proposition 3.3-(iii), one also has $\underline{b}_{1}=-\infty$ and $\bar{b}_{2}=\infty$.

(ii) Define

$$
\begin{array}{ll}
\zeta_{1}(y):=\sup \left\{x \in \mathbb{R}:-\alpha V_{x}(x, y)-f_{y}(x, y)-\rho K \geq 0\right\}, & y \in \mathbb{R}, \\
\zeta_{2}(y):=\inf \left\{x \in \mathbb{R}:-\alpha V_{x}(x, y)-f_{y}(x, y)+\rho K \leq 0\right\}, & y \in \mathbb{R} .
\end{array}
$$

Then, for any $y \in \mathbb{R}$, we have

$$
g_{1}(y)<\zeta_{1}(y)<\zeta_{2}(y)<g_{2}(y)
$$

Proof Proof of $(i)$. Here we show that $\lim _{x \downarrow-\infty} b_{1}(x)=\infty$. The fact that $\lim _{x \uparrow \infty} b_{2}(x)=-\infty$ can be proved by similar arguments. We argue by contradiction assuming $\bar{b}_{1}:=\lim _{x \downarrow-\infty} b_{1}(x)<\infty$. Take $y_{o}>\bar{b}_{1}$, so that $\tau^{\star}=\tau^{\star}\left(x, y_{o}\right)=\infty$ for all $x \in \mathbb{R}$, the latter being the stopping time defined in (3.3). Then, take $x_{o}<g_{2}\left(y_{o}\right)$ such that $\left(x_{o}, y_{o}\right) \in \mathcal{C}$. Clearly, every $x<x_{o}$ belongs to $\mathcal{C}$, and therefore, by the representation (4.15), we obtain that it must be $B\left(y_{o}\right)=0$; indeed, otherwise, by taking limits as $x \rightarrow-\infty$ and using (4.1), we would contradict Proposition 2.4. Moreover, since for any $y \in \mathbb{R}$ one has $\psi_{x}(x, \alpha y) \rightarrow 0$ when $x \rightarrow-\infty$ (cf. (4.1)), we then have by dominated convergence 
$\lim _{x \rightarrow-\infty} V_{x}\left(x, y_{0}\right)=\lim _{x \rightarrow-\infty} \widehat{V}_{x}\left(x, y_{o}\right)=\lim _{x \rightarrow-\infty} \mathrm{E}\left[\int_{0}^{\infty} e^{-\rho t} f_{x}\left(X_{t}^{x, y_{o}}, y_{o}\right) \mathrm{d} t\right]=-\infty$.

Now, setting

$$
\hat{\sigma}_{x}:=\inf \left\{t \geq 0: X_{t}^{x, y_{o}} \geq x_{o}\right\}
$$

for $x<x_{o}$, we have by monotonicity of $f_{y}(\cdot, y)$ (cf. Assumption 2.2-(iv))

$$
\begin{aligned}
-K & <V_{y}\left(x, y_{o}\right)=\inf _{\sigma \in \mathcal{T}} \mathrm{E}\left[\int_{0}^{\sigma} e^{-\rho t}\left(\alpha V_{x}\left(X_{t}^{x, y_{o}}, y_{o}\right)+f_{y}\left(X_{t}^{x, y_{o}}, y_{o}\right)\right) \mathrm{d} t+e^{-\rho \sigma} K\right] \\
& \leq \mathrm{E}\left[\int_{0}^{\hat{\sigma}_{x}} e^{-\rho t}\left(\alpha V_{x}\left(X_{t}^{x, y_{o}}, y_{o}\right)+f_{y}\left(x_{o}, y_{o}\right)\right) \mathrm{d} t+K\right] .
\end{aligned}
$$

The latter implies

$$
\begin{aligned}
2 K+\frac{\left|f_{y}\left(x_{o}, y_{o}\right)\right|}{\rho} & \geq-\alpha \mathrm{E}\left[\int_{0}^{\hat{\sigma}_{x}} e^{-\rho t} V_{x}\left(X_{t}^{x, y_{o}}, y_{o}\right) \mathrm{d} t\right] \\
& =-\alpha \mathrm{E}\left[\int_{0}^{\hat{\sigma}_{x}} e^{-\rho t} V_{x}\left(x+\alpha y_{o} t+\eta W_{t}, y_{o}\right) \mathrm{d} t\right]
\end{aligned}
$$

Hence, letting $x \downarrow-\infty$, using (5.1), and invoking the dominated convergence theorem we get a contradiction.

Proof of (ii). The fact that for any $y \in \mathbb{R}$ we have $g_{1}(y) \leq \zeta_{1}(y)$ and $g_{2}(y) \geq \zeta_{2}(y)$ can be obtained as in the proof of item (ii) of Proposition 6.1 in [21], by employing the proved regularity of $V_{y}(\cdot, y)$ and the semiharmonic characterization of [38] (see Eqs. (2.27)-(2.29) therein, suitably adjusted to take care of the integral term appearing in (3.2)). Moreover, $\zeta_{1}(y)<\zeta_{2}(y)$ for any $y \in \mathbb{R}$ by definition. It thus remains to show that one actually has $g_{1}(y)<\zeta_{1}(y)$ and $g_{2}(y)>\zeta_{2}(y)$ for any $y \in \mathbb{R}$.

We only prove that $g_{2}(y)>\zeta_{2}(y)$ for any $y \in \mathbb{R}$, as the other case can be treated similarly. Suppose that there exists some $y_{o}$ such that $g_{2}\left(y_{o}\right)=\zeta_{2}\left(y_{o}\right)$. Then $V_{y}\left(\zeta_{2}\left(y_{o}\right), y_{o}\right)=K$. Let now $\tau^{\star}:=\tau^{\star}\left(\zeta_{2}\left(y_{o}\right), y_{o}\right)$ be the optimal stopping time for the sup player when the Dynkin game (3.2) starts at time zero from the point $\left(\zeta_{2}\left(y_{o}\right), y_{o}\right)$, and for $\varepsilon>0$ define

$$
q_{\varepsilon}:=q_{\varepsilon}\left(\zeta_{2}\left(y_{o}\right), y_{o}\right):=\inf \left\{t \geq 0: X_{t}^{\zeta_{2}\left(y_{o}\right), y_{o}} \geq \zeta_{2}\left(y_{o}\right)+\varepsilon\right\}
$$

Then by using that $f_{y}\left(\cdot, y_{o}\right)+\alpha V_{x}\left(\cdot, y_{o}\right)$ is nondecreasing and locally Lipschitz by Assumption 2.2-(iii) and Proposition 2.4(iii), we have from (3.2) for some constant $C\left(y_{o}\right)>0$

$$
K=V_{y}\left(\zeta_{2}\left(y_{o}\right), y_{o}\right) \leq \mathrm{E}\left[\int_{0}^{\tau^{\star} \wedge q_{\varepsilon}} e^{-\rho t}\left(f_{y}+\alpha V_{x}\right)\left(X_{s}^{\zeta_{2}\left(y_{o}\right), y_{o}}, y_{o}\right) \mathrm{d} s\right]
$$




$$
\begin{aligned}
& +\mathrm{E}\left[K e^{-\rho q_{\varepsilon}} \mathbb{1}_{\left\{\tau^{\star}>q_{\varepsilon}\right\}}-K e^{-\rho \tau^{\star}} \mathbb{1}_{\left\{\tau^{\star}<q_{\varepsilon}\right\}}\right] \\
\leq & \left(f_{y}+\alpha V_{x}\right)\left(\zeta_{2}\left(y_{o}\right)+\varepsilon, y_{o}\right) \frac{1}{\rho} \mathrm{E}\left[1-e^{-\rho\left(\tau^{\star} \wedge q_{\varepsilon}\right)}\right] \\
& +\mathrm{E}\left[K e^{-\rho q_{\varepsilon}} \mathbb{1}_{\left\{\tau^{\star}>q_{\varepsilon}\right\}}-K e^{-\rho \tau^{\star}} \mathbb{1}_{\left\{\tau^{\star}<q_{\varepsilon}\right\}}\right] \\
\leq & \frac{1}{\rho}\left[\left(f_{y}+\alpha V_{x}\right)\left(\zeta_{2}\left(y_{o}\right), y_{o}\right)+\varepsilon C\left(y_{o}\right)\right] \mathrm{E}\left[1-e^{-\rho\left(\tau^{\star} \wedge q_{\varepsilon}\right)}\right] \\
& +\mathrm{E}\left[K e^{-\rho q_{\varepsilon}} \mathbb{1}_{\left\{\tau^{\star}>q_{\varepsilon}\right\}}-K e^{-\rho \tau^{\star}} \mathbb{1}_{\left\{\tau^{\star}<q_{\varepsilon}\right\}}\right] .
\end{aligned}
$$

Using now that, by definition of $\zeta_{2}$, it must be $\left(f_{y}+\alpha V_{x}\right)\left(\zeta_{2}\left(y_{o}\right), y_{o}\right)=\rho K$, and rearranging terms, we get that

$$
0 \leq \frac{\varepsilon C\left(y_{o}\right)}{\rho} \mathrm{E}\left[1-e^{-\rho\left(\tau^{\star} \wedge q_{\varepsilon}\right)}\right]-2 K \mathrm{E}\left[e^{-\rho \tau^{\star}} \mathbb{1}_{\left\{\tau^{\star}<q_{\varepsilon}\right\}}\right]
$$

Notice now that (cf. eq. (4.3) in [15], among others)

$$
\mathrm{E}\left[e^{-\rho \tau^{\star}} \mathbb{1}_{\left\{\tau^{\star}<q_{\varepsilon}\right\}}\right]=\frac{\psi\left(\zeta_{2}\left(y_{o}\right), \alpha y_{o}\right) \varphi\left(\zeta_{2}\left(y_{o}\right)+\varepsilon, \alpha y_{o}\right)-\psi\left(\zeta_{2}\left(y_{o}\right)+\varepsilon, \alpha y_{o}\right) \varphi\left(\zeta_{2}\left(y_{o}\right), \alpha y_{o}\right)}{\psi\left(g_{1}\left(y_{o}\right), \alpha y_{o}\right) \varphi\left(\zeta_{2}\left(y_{o}\right)+\varepsilon, \alpha y_{o}\right)-\psi\left(\zeta_{2}\left(y_{o}\right)+\varepsilon, \alpha y_{o}\right) \varphi\left(g_{1}\left(y_{o}\right), \alpha y_{o}\right)}
$$

and

$$
\mathrm{E}\left[e^{-\rho q_{\varepsilon}} \mathbb{1}_{\left\{\tau^{\star}>q_{\varepsilon}\right\}}\right]=\frac{\psi\left(g_{1}\left(y_{o}\right), \alpha y_{o}\right) \varphi\left(\zeta_{2}\left(y_{o}\right), \alpha y_{o}\right)-\psi\left(\zeta_{2}\left(y_{o}\right), \alpha y_{o}\right) \varphi\left(g_{1}\left(y_{o}\right), \alpha y_{o}\right)}{\psi\left(g_{1}\left(y_{o}\right), \alpha y_{o}\right) \varphi\left(\zeta_{2}\left(y_{o}\right)+\varepsilon, \alpha y_{o}\right)-\psi\left(\zeta_{2}\left(y_{o}\right)+\varepsilon, \alpha y_{o}\right) \varphi\left(g_{1}\left(y_{o}\right), \alpha y_{o}\right)} .
$$

Then, because

$$
1-e^{-\rho\left(\tau^{\star} \wedge q_{\varepsilon}\right)}=1-e^{-\rho \tau^{*}} \mathbb{1}_{\left\{\tau^{\star}<q_{\varepsilon}\right\}}-e^{-\rho q_{\varepsilon}} \mathbb{1}_{\left\{\tau^{\star}>q_{\varepsilon}\right\}},
$$

using the last two formulas in (5.4) and performing a first-order Taylor's expansion around $\varepsilon=0$ of the terms on the right-hand side of (5.4), one finds that the first term on the right-hand side of (5.4) is positive and converges to zero as $\varepsilon \downarrow 0$ with order $\varepsilon^{2}$, while the second term is negative and converges to zero with order $\varepsilon$. We thus reach a contradiction in (5.4) for $\varepsilon$ small enough, and therefore it cannot exist $y_{o}$ at which $g_{2}\left(y_{o}\right)=\zeta_{2}\left(y_{o}\right)$.

The next result readily follows from Proposition 5.3-(i).

Corollary 5.4 Let Assumption 5.1-(i) hold. Then the functions $g_{1}, g_{2}$ defined in (3.8) are finite.

We now move on by proving that the boundaries $b_{1}$ and $b_{2}$ are strictly monotone. This will in turn imply that their inverses $g_{1}$ and $g_{2}$ are actually continuous.

Proposition 5.5 Let Assumption 5.1 hold. Then the functions $b_{1}, b_{2}$ are strictly decreasing. 
Proof We prove the claim only for $b_{1}$, since analogous arguments apply to prove it for $b_{2}$.

Case (a). We assume here that item (a) of Assumption 5.1-(iii) holds, i.e. that $x \mapsto f_{y}(x, y)$ is strictly increasing for any $y \in \mathbb{R}$. By Proposition 4.5 , we can differentiate the first line of (4.15) with respect to $y$ and get by Proposition 4.4-(i) that $V_{y}$ solves inside $\mathcal{C}$ the equation

$$
\frac{1}{2} \eta^{2} V_{y x x}(x, y)+\alpha y V_{y x}(x, y)-\rho V_{y}(x, y)=-f_{y}(x, y)-\alpha V_{x}(x, y) .
$$

By continuity, (5.5) also holds on $\overline{\mathcal{C}}$, i.e.

$$
\begin{gathered}
\frac{1}{2} \eta^{2} V_{y x x}(x, y)+\alpha y V_{y x}(x, y)-\rho V_{y}(x, y) \\
=-f_{y}(x, y)-\alpha V_{x}(x, y), \quad \forall(x, y) \in \overline{\mathcal{C}} .
\end{gathered}
$$

In particular it holds on $\partial^{1} \mathcal{C}:=\overline{\mathcal{C}} \cap \mathcal{I}$. Assume now, by contradiction, that the boundary $b_{1}$ is constant on $\left(x_{o}, x_{o}+\varepsilon\right)$, for some $x_{o} \in \mathbb{R}$ and some $\varepsilon>0$. Then, setting $y_{o}:=b_{1}\left(x_{o}\right)$, we will have $V_{y x x}\left(\cdot, y_{o}\right)=V_{y x}\left(\cdot, y_{o}\right)=0$ and $V_{y}\left(\cdot, y_{o}\right)=-K$ on $\left(x_{o}, x_{o}+\varepsilon\right)$. Hence, we obtain from (5.5) that

$$
-\rho K=f_{y}\left(x, y_{o}\right)+\alpha V_{x}\left(x, y_{o}\right), \quad \forall x \in\left(x_{o}, x_{o}+\varepsilon\right),
$$

and thus

$$
-f_{y x}\left(x, y_{o}\right)=\alpha V_{x x}\left(x, y_{o}\right), \quad \forall x \in\left(x_{o}, x_{o}+\varepsilon\right) .
$$

But now $\alpha V_{x x}\left(x, y_{o}\right) \geq 0$ for any $x \in\left(x_{o}, x_{o}+\varepsilon\right)$ by convexity of $V\left(\cdot, y_{o}\right)$, while, by assumption, $f_{y x}$ must be strictly positive on a subset of $\left(x_{o}, x_{o}+\varepsilon\right)$ with positive measure. Hence a contradiction is reached.

Case (b). We assume here that item (b) of Assumption 5.1-(iii) holds, i.e. that $f_{y x} \equiv 0$ and that $f(\cdot, y)$ is strictly convex for any $y \in \mathbb{R}$. In such a case the claim can be proved by employing the same arguments of the proof of Proposition 6.3 in [21].

From the above result, the following corollary is immediate.

Corollary 5.6 Let Assumption 5.1 hold. Then the functions $g_{1}, g_{2}$ defined in (3.8) are continuous.

The next result will be of fundamental importance to show the local Lipschitz property of $g_{i}, i=1,2$ via a suitable application of the implicit function theorem (cf. Proposition 5.8 below).

Proposition 5.7 Let Assumption 5.1 hold. Then there exists

$$
\ell\left(x_{o}, y_{o}\right):=\lim _{\substack{(x, y) \rightarrow\left(x_{o}, y_{o}\right) \\(x, y) \in \mathcal{C}}} V_{y x x}(x, y) \quad \forall\left(x_{o}, y_{o}\right) \in \partial \mathcal{C},
$$


and one has $\ell\left(x_{o}, y_{o}\right) \neq 0$ for any $\left(x_{o}, y_{o}\right) \in \partial \mathcal{C}$.

Proof We provide the proof only for any $\left(x_{o}, y_{o}\right) \in \partial^{2} \mathcal{C}:=\overline{\mathcal{C}} \cap \mathcal{D}$, as the other case can be treated similarly.

First of all, we notice that the limit in (5.9) exists since, by Proposition 4.5, the function $V: \mathcal{C} \rightarrow \mathbb{R}$ can be differentiated twice with respect to $x$ and once with respect to $y$ with continuity up to the boundary $\partial \mathcal{C}$.

Case (a). We assume here that item (a) of Assumption 5.1-(iii) holds, i.e. that $x \mapsto f_{y}(x, y)$ is strictly increasing for any $y \in \mathbb{R}$. Suppose, by contradiction, that for some $y_{o} \in \mathbb{R}$ one has

$$
\lim _{(x, y) \rightarrow\left(g_{2}\left(y_{o}\right), y_{o}\right)} V_{y x x}(x, y)=0 .
$$

Then taking limits as $(x, y) \rightarrow\left(g_{2}\left(y_{o}\right), y_{o}\right)$ for $(x, y) \in \mathcal{C}$ in (4.13) we find, using that $V_{y x}\left(g_{2}\left(y_{o}\right), y_{o}\right)=0$ by Proposition 4.7 and that $V_{y}\left(g_{2}\left(y_{o}\right), y_{o}\right)=K$,

$$
-\rho K+f_{y}\left(g_{2}\left(y_{o}\right), y_{o}\right)=-\alpha V_{x}\left(g_{2}\left(y_{o}\right), y_{o}\right) .
$$

Since $g_{2}\left(y_{o}\right)>\zeta_{2}\left(y_{o}\right)$ by Proposition 5.3, and by definition of $\zeta_{2}$, it must be

$$
-\rho K+f_{y}\left(x, y_{o}\right)=-\alpha V_{x}\left(x, y_{o}\right) \quad \forall x \in\left(\zeta_{2}\left(y_{o}\right), g_{2}\left(y_{o}\right)\right)
$$

which also implies that $-\alpha V_{x x}\left(x, y_{o}\right)=f_{y x}\left(x, y_{o}\right)$ for any $x \in\left(\zeta_{2}\left(y_{o}\right), g_{2}\left(y_{o}\right)\right)$. We then conclude as in Case (a) of the proof of Proposition 5.5.

Case (b). We assume here that item (b) of Assumption 5.1-(iii) holds, which implies that there exists $q$ such that $f_{y}(x, y)=q(y)$ for any $(x, y) \in \mathbb{R}^{2}$. Suppose again, with the aim of reaching a contradiction, that for some $y_{o} \in \mathbb{R}$ one has (5.10). Then taking limits as $(x, y) \rightarrow\left(g_{2}\left(y_{o}\right), y_{o}\right)$ for $(x, y) \in \mathcal{C}$ in (4.13) we find, using that $V_{y x}\left(g_{2}\left(y_{o}\right), y_{o}\right)=0$ by Proposition 4.7 and that $V_{y}\left(g_{2}\left(y_{o}\right), y_{o}\right)=K$,

$$
-\rho K+q\left(y_{o}\right)=-\alpha V_{x}\left(g_{2}\left(y_{o}\right), y_{o}\right)
$$

As before, because $g_{2}\left(y_{o}\right)>\zeta_{2}\left(y_{o}\right)$ by Proposition 5.3, and by definition of $\zeta_{2}$, it must be

$$
-\rho K+q\left(y_{o}\right)=-\alpha V_{x}\left(x, y_{o}\right) \quad \forall x \in\left(\zeta_{2}\left(y_{o}\right), g_{2}\left(y_{o}\right)\right)
$$

that is, $V$ is an affine function of $x$ in that interval. However, using the latter and (4.13), we also have

$$
\frac{1}{\alpha} \alpha y_{o}\left(\rho K-q\left(y_{o}\right)\right)-\rho V\left(x, y_{o}\right)=-f\left(x, y_{o}\right) \quad \forall x \in\left(\zeta_{2}\left(y_{o}\right), g_{2}\left(y_{o}\right)\right),
$$

and we reach a contradiction since $f\left(\cdot, y_{o}\right)$ is strictly convex by assumption, while $V\left(\cdot, y_{o}\right)$ is affine. 
Thanks to the previous analysis we are finally able to prove the aforementioned LIpschitz regularity of $g_{1}$ and $g_{2}$.

Proposition 5.8 Let Assumption 5.1 hold. Then the functions $g_{1}, g_{2}$ are locally Lipschitz.

Proof Define the function

$$
\bar{V}(x, y):=A(y) \psi(x, \alpha y)+B(y) \varphi(x, \alpha y)+\widehat{V}(x, y), \quad(x, y) \in \mathbb{R}^{2},
$$

where $A, B$ are the functions of Proposition 4.5. Then, one clearly has that $\bar{V} \in$ $C^{2,1}\left(\mathbb{R}^{2} ; \mathbb{R}\right)$ (since, by Sobolev embedding, $A$ and $B$ belong to $C_{\text {loc }}^{1, \text { Lip }}(\mathbb{R} ; \mathbb{R})$ ), and $\bar{V}=V$ in $\mathbb{R}^{2} \cap \overline{\mathcal{C}}$. Moreover, the mixed derivative $\bar{V}_{y x}$ exists and is continuous, and standard differentiation yield

$$
\begin{aligned}
\bar{V}_{y x}(x, y)= & A_{y}(y) \psi_{x}(x, \alpha y)+B_{y}(y) \varphi_{x}(x, \alpha y)+\alpha\left(A(y) \psi_{z x}(x, \alpha y)\right. \\
& \left.+B(y) \varphi_{z x}(x, \alpha y)\right)+\widehat{V}_{y x}(x, y) .
\end{aligned}
$$

Since $A_{y}$ and $B_{y}$ are locally Lipschitz by Proposition 4.5 , and $\psi$ and $\varphi$ are smooth (cf. (4.1)), we deduce that $\bar{V}_{y x}(x, \cdot)$ is locally Lipschitz.

Let now $y_{o} \in \mathbb{R}$. Then, for any given $x_{o} \in \mathbb{R}$ such that $\left(x_{o}, y_{o}\right) \in \partial \mathcal{C}$, we know by Proposition 5.7 that $\bar{V}_{y x x}\left(x_{o}, y_{o}\right) \neq 0$, while $\bar{V}_{y x}\left(x_{o}, y_{o}\right)=0$. Therefore, the implicit function theorem (see, e.g., the Corollary at p. 256 in [14] or Theorem 3.1 in [36]) implies that, for any $i=1,2$ and for suitable $\delta, \delta^{\prime}>0$, there exists a unique continuous function $\bar{g}_{i}:\left(y_{o}-\delta, y_{o}+\delta\right) \rightarrow\left(x_{o}-\delta^{\prime}, x_{o}+\delta^{\prime}\right)$ such that $\bar{V}_{y x}\left(\bar{g}_{i}(y), y\right)=0$ in $\left(y_{o}-\delta, y_{o}+\delta\right)$. Also, the aforementioned properties of $\bar{V}_{y x y}$ and $\bar{V}_{y x x}$ imply that there exists $C\left(y_{o}\right)>0$ such that

$$
\left|\bar{g}_{i}\left(y_{2}\right)-\bar{g}_{i}\left(y_{1}\right)\right| \leq C\left(y_{o}\right)\left|y_{2}-y_{1}\right|, \quad \forall y_{1}, y_{2} \in\left(y_{o}-\delta, y_{o}+\delta\right) .
$$

Recalling now that $\bar{V}_{y x}\left(g_{i}(y), y\right)=0$, we can identify $\bar{g}_{i}=g_{i}, i=1,2$, in $\left(y_{o}-\delta, y_{o}+\delta\right)$ and therefore $g_{i}$ is locally Lipschitz therein. Given the arbitrariness of the point $\left(x_{o}, y_{o}\right)$ the proof is complete.

\section{A System of Differential Equations for the Free Boundaries}

In this section we derive a first-order system of nonlinear differential equations for the free boundaries $g_{1}$ and $g_{2}$, i.e. we will be able to write

$$
\left\{\begin{array}{l}
g_{1}^{\prime}(y)=G_{1}\left(g_{1}(y), g_{2}(y), y\right), \\
g_{2}^{\prime}(y)=G_{2}\left(g_{1}(y), g_{2}(y), y\right),
\end{array}\right.
$$

for some explicitly determined maps $G_{1}, G_{2}$, whose regularity will allow also to establish a $C^{1, \text { Lip }}$ regularity for $g_{1}, g_{2}$. To the best of our knowledge, for a two-dimensional 
degenerate singular stochastic control problem with interconnected dynamics as ours, a similar result appears here for the first time.

We first move on by establishing four equations relating $g_{1}, g_{2}$ and $A, B$. Recall (4.6), (4.7), and (4.9). We also denote by $p$ the transition density of $X^{x, y}$ with respect to the speed measure; then, letting $A \mapsto \mathrm{P}_{t}(x, A, y), A \in \mathcal{B}(\mathbb{R}), t>0$ and $y \in \mathbb{R}$, be the probability of starting at time 0 from level $x \in \mathbb{R}$ and reaching the set $A \in \mathcal{B}(\mathbb{R})$ in $t$ units of time, we have (cf., e.g., p. 13 in [6])

$$
\mathrm{P}_{t}(x, A, y)=\int_{A} p(t, x, z, y) m_{x}(z, \alpha y) \mathrm{d} z .
$$

The density $p$ can be taken positive, jointly continuous in all variables and symmetric (i.e. $p(t, x, z, y)=p(t, z, x, y))$.

Theorem 6.1 Let Assumption 5.1 hold. Recall (4.1), (4.15), and for any $(x, y) \in \mathbb{R}^{2}$ define

$$
H(x, y):=f_{y}(x, y)+\alpha V_{x}(x, y) .
$$

Then, the free boundaries $g_{1}$ and $g_{2}$ as in (3.8), and the coefficients $A$ and $B$ are such that

$$
\begin{aligned}
& 0=\int_{g_{1}(y)}^{g_{2}(y)} \psi(z, \alpha y) H(z, y) m_{x}(z, \alpha y) \mathrm{d} z-K \frac{\psi_{x}\left(g_{1}(y), \alpha y\right)}{S_{x}\left(g_{1}(y), \alpha y\right)}-K \frac{\psi_{x}\left(g_{2}(y), \alpha y\right)}{S_{x}\left(g_{2}(y), \alpha y\right)}, \\
& 0=\int_{g_{1}(y)}^{g_{2}(y)} \varphi(z, \alpha y) H(z, y) m_{x}(z, \alpha y) \mathrm{d} z-K \frac{\varphi_{x}\left(g_{1}(y), \alpha y\right)}{S_{x}\left(g_{1}(y), \alpha y\right)}-K \frac{\varphi_{x}\left(g_{2}(y), \alpha y\right)}{S_{x}\left(g_{2}(y), \alpha y\right)}, \\
& A^{\prime}(y) \psi_{x}\left(g_{1}(y), \alpha y\right)+B^{\prime}(y) \varphi_{x}\left(g_{1}(y), \alpha y\right)+\widehat{V}_{y x}\left(g_{1}(y), y\right) \\
& \quad+\alpha\left[A(y) \psi_{z x}\left(g_{1}(y), \alpha y\right)+B(y) \varphi_{z x}\left(g_{1}(y), \alpha y\right)\right]=0 \\
& A^{\prime}(y) \psi_{x}\left(g_{2}(y), \alpha y\right)+B^{\prime}(y) \varphi_{x}\left(g_{2}(y), \alpha y\right) \widehat{V}_{y x}\left(g_{2}(y), y\right) \\
& \quad+\alpha\left[A(y) \psi_{z x}\left(g_{2}(y), \alpha y\right)+B(y) \varphi_{z x}\left(g_{2}(y), \alpha y\right)\right]=0 .
\end{aligned}
$$

Proof To obtain Eqs. (6.3) and (6.4) we exploit the proved regularity of $V(\cdot, y)$ (cf. Propositions 2.4, 4.7, and Lemma 4.8) in order to follow the proof of Theorem 6.5 in [21]. This is based on an application of the local time-space calculus of [37] to the process $\left(e^{-\rho s} V_{y}\left(X_{s}, y\right)\right)_{s \geq 0}$ and the use of the Green function (4.9), the transition probability (6.1), and Fubini's theorem. Alternatively (and equivalently) they can be derived from (4.15) by imposing that $V_{y}(\cdot, y)$ and $V_{y x}(\cdot, y)$ are continuous on $\partial \mathcal{C}$ and proceeding via the more analytical direct approach of the proof of Proposition 5.5 in [20]. In particular, the second-order smooth fit $V_{y x}\left(g_{i}(y), y\right)=0, i=1,2$, easily gives (6.5) and (6.6). 
Corollary 6.2 Let Assumption 5.1 hold. For $z \in \mathbb{R}$ define

$$
\lambda(z):=\sqrt{z^{2}+2 \rho \eta^{2}},
$$

and for $y \in \mathbb{R}, i, j=1,2, j \neq i$,

$$
\begin{aligned}
\gamma_{i}(y):= & -\frac{2}{\eta^{2}} \int_{g_{1}(y)}^{g_{2}(y)} e^{-r_{j}(\alpha y) u}\left(f_{y}(u, y)+\alpha \widehat{V}_{x}(u, y)\right) \mathrm{d} u \\
& +K r_{i}(\alpha y)\left(e^{-r_{j}(\alpha y) g_{1}(y)}+e^{-r_{j}(\alpha y) g_{2}(y)}\right) .
\end{aligned}
$$

Furthermore, for any $\left(x_{1}, x_{2}, y\right) \in\left\{\left(x_{1}, x_{2}, y\right) \in \mathbb{R}^{3}: x_{1} \neq x_{2}\right\}$, define

$$
\begin{aligned}
M\left(x_{1}, x_{2}, y\right):= & r_{2}(\alpha y)\left(e^{r_{2}(\alpha y) x_{1}} \widehat{V}_{y x}\left(x_{2}, y\right)-e^{r_{2}(\alpha y) x_{2}} \widehat{V}_{y x}\left(x_{1}, y\right)\right) \\
& +\alpha A(y) r_{2}^{\prime}(\alpha y) r_{2}(\alpha y)\left(e^{r_{1}(\alpha y) x_{2}+r_{2}(\alpha y) x_{1}}\left(r_{2}(\alpha y) x_{2}+1\right)\right. \\
& \left.-e^{r_{1}(\alpha y) x_{1}+r_{2}(\alpha y) x_{2}}\left(r_{1}(\alpha y) x_{1}+1\right)\right) \\
& +\alpha B(y) r_{2}^{2}(\alpha y) r_{2}^{\prime}(\alpha y) e^{r_{2}(\alpha y)\left(x_{1}+x_{2}\right)}\left(x_{2}-x_{1}\right)
\end{aligned}
$$

and

$$
\begin{aligned}
N\left(x_{1}, x_{2}, y\right):= & r_{1}(\alpha y)\left(e^{r_{1}(\alpha y) x_{2}} \widehat{V}_{y x}\left(x_{1}, y\right)-e^{r_{1}(\alpha y) x_{1}} \widehat{V}_{y x}\left(x_{2}, y\right)\right) \\
& +\alpha B(y) r_{2}^{\prime}(\alpha y) r_{1}(\alpha y)\left(e^{r_{1}(\alpha y) x_{2}+r_{2}(\alpha y) x_{1}}\left(r_{2}(\alpha y) x_{1}+1\right)\right. \\
& \left.-e^{r_{1}(\alpha y) x_{1}+r_{2}(\alpha y) x_{2}}\left(r_{2}(\alpha y) x_{2}+1\right)\right) \\
& -\alpha A(y) r_{1}^{2}(\alpha y) r_{1}^{\prime}(\alpha y) e^{r_{1}(\alpha y)\left(x_{1}+x_{2}\right)}\left(x_{2}-x_{1}\right) .
\end{aligned}
$$

Then, one has

$$
\begin{aligned}
A(y)= & -\frac{r_{2}(\alpha y) \eta^{2} \lambda(\alpha y)}{8 \alpha \rho} \\
& {\left[\frac{\gamma_{1}(y)\left(e^{-\frac{2}{\eta^{2}} \lambda(\alpha y) g_{1}(y)}-e^{-\frac{2}{\eta^{2}} \lambda(\alpha y) g_{2}(y)}\right)-\frac{2 \lambda(\alpha y) \gamma_{2}(y)}{\eta^{2}}\left(g_{2}(y)-g_{1}(y)\right)}{\sinh ^{2}\left(\frac{\lambda(\alpha y)}{\eta^{2}}\left(g_{2}(y)-g_{1}(y)\right)\right)-\left(\frac{\lambda(\alpha y)}{\eta^{2}}\left(g_{2}(y)-g_{1}(y)\right)\right)^{2}}\right], } \\
B(y)= & \left.-\frac{r_{1}(\alpha y) \eta^{2} \lambda(\alpha y)}{8 \alpha \rho}\right] \\
& {\left[\frac{\gamma_{2}(y)\left(e^{\frac{2}{\eta^{2}} \lambda(\alpha y) g_{2}(y)}-e^{\frac{2}{\eta^{2}} \lambda(\alpha y) g_{1}(y)}\right)-\frac{2 \lambda(\alpha y) \gamma_{1}(y)}{\eta^{2}}\left(g_{2}(y)-g_{1}(y)\right)}{\sinh ^{2}\left(\frac{\lambda(\alpha y)}{\eta^{2}}\left(g_{2}(y)-g_{1}(y)\right)\right)-\left(\frac{\lambda(\alpha y)}{\eta^{2}}\left(g_{2}(y)-g_{1}(y)\right)\right)^{2}}\right], }
\end{aligned}
$$


as well as

$$
\begin{aligned}
& A^{\prime}(y)=\frac{\eta^{2}}{2 \rho} e^{\frac{2 \alpha y}{\eta^{2}} g_{1}(y)}\left[\frac{M\left(g_{1}(y), g_{2}(y), y\right)}{e^{r_{1}(\alpha y)\left(g_{2}(y)-g_{1}(y)\right)}-e^{r_{2}(\alpha y)\left(g_{2}(y)-g_{1}(y)\right)}}\right], \\
& B^{\prime}(y)=\frac{\eta^{2}}{2 \rho} e^{\frac{2 \alpha y}{\eta^{2}} g_{1}(y)}\left[\frac{N\left(g_{1}(y), g_{2}(y), y\right)}{e^{r_{1}(\alpha y)\left(g_{2}(y)-g_{1}(y)\right)}-e^{r_{2}(\alpha y)\left(g_{2}(y)-g_{1}(y)\right)}}\right] .
\end{aligned}
$$

Proof In order to derive (6.9) and (6.10), notice that, given $g_{1}$ and $g_{2}$, and exploiting (4.15), one has from (6.3) and (6.4) that $A$ and $B$ solve the linear system

$$
\begin{aligned}
A(y) & {\left[\alpha \int_{g_{1}(y)}^{g_{2}(y)} \psi(z, \alpha y) \psi_{x}(z, \alpha y) m_{x}(z, \alpha y) \mathrm{d} z\right] } \\
& +B(y)\left[\alpha \int_{g_{1}(y)}^{g_{2}(y)} \psi(z, \alpha y) \varphi_{x}(z, \alpha y) m_{x}(z, \alpha y) \mathrm{d} z\right] \\
= & K\left[\frac{\psi_{x}\left(g_{1}(y), \alpha y\right)}{S_{x}\left(g_{1}(y), \alpha y\right)}+\frac{\psi_{x}\left(g_{2}(y), \alpha y\right)}{S_{x}\left(g_{2}(y), \alpha y\right)}\right] \\
& -\int_{g_{1}(y)}^{g_{2}(y)} \psi(z, \alpha y)\left(f_{y}(z, y)+\alpha \widehat{V}_{x}(z, y)\right) m_{x}(z, \alpha y) \mathrm{d} z \\
A(y) & {\left[\alpha \int_{g_{1}(y)}^{g_{2}(y)} \varphi(z, \alpha y) \psi_{x}(z, \alpha y) m_{x}(z, \alpha y) \mathrm{d} z\right] } \\
& +B(y)\left[\alpha \int_{g_{1}(y)}^{g_{2}(y)} \varphi(z, \alpha y) \varphi_{x}(z, \alpha y) m_{x}(z, \alpha y) \mathrm{d} z\right] \\
= & K\left[\frac{\varphi_{x}\left(g_{1}(y), \alpha y\right)}{S_{x}\left(g_{1}(y), \alpha y\right)}+\frac{\varphi_{x}\left(g_{2}(y), \alpha y\right)}{S_{x}\left(g_{2}(y), \alpha y\right)}\right] \\
& \int_{g_{1}(y)}^{g_{2}(y)} \varphi(z, \alpha y)\left(f_{y}(z, y)+\alpha \widehat{V}_{x}(z, y)\right) m_{x}(z, \alpha y) \mathrm{d} z .
\end{aligned}
$$

By using expressions for $\psi, \varphi, S_{x}$ and $m_{x}$ (cf. (4.1), (4.6) and (4.7)) one can explicitly evaluate the integrals appearing on the left-hand sides of (6.13) and (6.14). Then, solving the latter two equations with respect to $A$ and $B$ one finds after some simple but tedious algebra (6.9) and (6.10). Notice indeed that the denominator appearing in (6.9) and (6.10) is nonzero since $g_{1} \neq g_{2}$ and one has $\sinh ^{2}(z)-z^{2}>0$ for any $z \neq 0$.

In order to find (6.11) and (6.12) we solve (6.5) and (6.6) with respect to $A^{\prime}(y)$ and $B^{\prime}(y)$, and use (4.1), (4.2), (4.3), and (6.7).

Remark 6.3 In [21] a system of Eqs. like (6.3) and (6.4) has also been obtained (see eqs. (6.11) and (6.12) therein). However, in [21] the uncontrolled process is of OrnsteinUhlenbeck type and this made it not possible to determine explicit expressions for $A(y)$ and $B(y)$ as in (6.9) and (6.10) above. Indeed, the complex form of the functions $\psi$ and $\varphi$ associated to the Ornstein-Uhlenbeck process does not allow to conclude that the determinant of the coefficients' matrix arising when one tries to solve (the analogous of) (6.13) and (6.13) with respect to $A(y)$ and $B(y)$ is nonzero. 
We can now state the main result of this paper.

Theorem 6.4 Let $D:=\left\{\left(x_{1}, x_{2}, y\right) \in \mathbb{R}^{3}: x_{1} \neq x_{2}\right\}$. There exist explicitly computable ${ }^{1}$ functions $G_{i} \in C_{\text {loc }}^{0, \text { Lip }}(D ; \mathbb{R}), i=1,2$ such that

$$
\left\{\begin{array}{l}
g_{1}^{\prime}(y)=G_{1}\left(g_{1}(y), g_{2}(y), y\right) \\
g_{2}^{\prime}(y)=G_{2}\left(g_{1}(y), g_{2}(y), y\right) .
\end{array}\right.
$$

In particular, $g_{i} \in C_{\text {loc }}^{1, \text { Lip }}(\mathbb{R} ; \mathbb{R})$ for $i=1,2$.

Proof Recall Theorem 4.5 and (6.2). In particular, for any $(x, y)$ such that $g_{1}(y) \leq$ $x \leq g_{2}(y)-$ i.e. for any $(x, y) \in \overline{\mathcal{C}}-$ we have by $(4.15)$

$$
V_{x}(x, y)=A(y) \psi_{x}(x, \alpha y)+B(y) \varphi_{x}(x, \alpha y)+\widehat{V}_{x}(x, y),
$$

with $A, B$ belonging to $W_{\text {loc }}^{2, \infty}(\mathbb{R} ; \mathbb{R})$. Defining then the function

$$
\bar{H}(x, y)=f_{y}(x, y)+\alpha\left(A(y) \psi_{x}(x, \alpha y)+B(y) \varphi_{x}(x, \alpha y)+\widehat{V}_{x}(x, y)\right), \quad(x, y) \in \mathbb{R}^{2},
$$

one has $\bar{H}=H$ on $\overline{\mathcal{C}}$.

Introduce now $\Phi_{i}: D \rightarrow \mathbb{R}$ defined as

$$
\begin{aligned}
\Phi_{1}\left(x_{1}, x_{2}, y\right):= & \int_{x_{1}}^{x_{2}} \psi(z, \alpha y) \bar{H}(z, y) m_{x}(z, \alpha y) \mathrm{d} z \\
& -K \rho \int_{-\infty}^{x_{1}} \psi(z, \alpha y) m_{x}(z, \alpha y) \mathrm{d} z-K \rho \int_{-\infty}^{x_{2}} \psi(z, \alpha y) m_{x}(z, \alpha y) \mathrm{d} z \\
\Phi_{2}\left(x_{1}, x_{2}, y\right):= & \int_{x_{1}}^{x_{2}} \varphi(z, \alpha y) \bar{H}(z, y) m_{x}(z, \alpha y) \mathrm{d} z \\
& +K \rho \int_{x_{2}}^{\infty} \varphi(z, \alpha y) m_{x}(z, \alpha y) \mathrm{d} z+K \rho \int_{x_{1}}^{\infty} \varphi(z, \alpha y) m_{x}(z, \alpha y) \mathrm{d} z .
\end{aligned}
$$

Observing that (cf. Chapter II in [6])

$$
\begin{aligned}
\frac{\psi_{x}(\cdot, \alpha y)}{S_{x}(\cdot, \alpha y)} & =\rho \int_{-\infty}^{\cdot} \psi(z, \alpha y) m_{x}(z, \alpha y) \mathrm{d} z \\
\frac{\varphi_{x}(\cdot, \alpha y)}{S_{x}(\cdot, \alpha y)} & =-\rho \int^{\infty} \varphi(z, \alpha y) m_{x}(z, \alpha y) \mathrm{d} z,
\end{aligned}
$$

one can readily see that, by (6.3)-(6.4), for any $y \in \mathbb{R}$ one has

$$
\Phi_{1}\left(g_{1}(y), g_{2}(y), y\right)=0 \text { and } \Phi_{2}\left(g_{1}(y), g_{2}(y), y\right)=0 .
$$

\footnotetext{
${ }^{1}$ Cf. Remark 6.5.
} 
Thanks to Assumption 2.2 and Theorem 4.5, one has that $\bar{H} \in C_{\text {loc }}^{1, \text { Lip }}\left(\mathbb{R}^{2} ; \mathbb{R}\right)$. Hence, for any $i=1,2$, the map $\left(x_{1}, x_{2}\right) \mapsto \Phi_{i}\left(x_{1}, x_{2}, y\right)$ belongs to $C^{2}(D ; \mathbb{R})$ for each $y \in \mathbb{R}$ and the map $y \mapsto \Phi_{i}\left(x_{1}, x_{2}, y\right)$ belongs to $C_{\text {loc }}^{1, \text { Lip }}(D ; \mathbb{R})$ for each $\left(x_{1}, x_{2}\right) \in \mathbb{R}^{2}$. Recalling Proposition 5.8 we can take the total derivative on both terms appearing in (6.18) we obtain for a.e. $y \in \mathbb{R}$ that

$$
\underbrace{\left(\begin{array}{l}
\frac{\partial \Phi_{1}}{\partial x_{1}}\left(g_{1}(y), g_{2}(y), y\right) \frac{\partial \Phi_{1}}{\partial x_{2}}\left(g_{1}(y), g_{2}(y), y\right) \\
\frac{\partial \Phi_{2}}{\partial x_{1}}\left(g_{1}(y), g_{2}(y), y\right) \frac{\partial \Phi_{2}}{\partial x_{2}}\left(g_{1}(y), g_{2}(y), y\right)
\end{array}\right)}_{=: \Lambda\left(g_{1}(y), g_{2}(y), y\right)}\left(\begin{array}{c}
g_{1}^{\prime}(y) \\
g_{2}^{\prime}(y)
\end{array}\right)=-\left(\begin{array}{l}
\frac{\partial \Phi_{1}}{\partial y}\left(g_{1}(y), g_{2}(y), y\right) \\
\frac{\partial \Phi_{2}}{\partial y}\left(g_{1}(y), g_{2}(y), y\right)
\end{array}\right) .
$$

The determinant of the matrix $\Lambda$, denoted by $|\Lambda|$, is given by

$$
\begin{aligned}
|\Lambda|\left(g_{1}(y), g_{2}(y), y\right)= & \left(\bar{H}\left(g_{1}(y), y\right)+K \rho\right)\left(\bar{H}\left(g_{2}(y), y\right)-K \rho\right) m_{x}\left(g_{1}(y), \alpha y\right) m_{x}\left(g_{2}(y), \alpha y\right) . \\
& \cdot\left(\psi\left(g_{2}(y), \alpha y\right) \varphi\left(g_{1}(y), \alpha y\right)-\psi\left(g_{1}(y), \alpha y\right) \varphi\left(g_{2}(y), \alpha y\right)\right) .
\end{aligned}
$$

We now aim at showing that $|\Lambda|\left(g_{1}(y), g_{2}(y), y\right)$ does not vanish for any $y \in \mathbb{R}$ under Assumption 5.1-(iii). On the one hand, if item (a) of that assumption holds, i.e. $x \mapsto f_{y}(x, y)$ is strictly increasing, then we have that $x \mapsto H(x, y)$ is such as well. Since $\bar{H}=H$ on $\overline{\mathcal{C}}$ and $g_{2}(y)>\zeta_{2}(y)>\zeta_{1}(y)>g_{1}(y)$ by Proposition 5.3-(ii), we have

$$
\bar{H}\left(g_{1}(y), y\right)+K \rho<0, \quad \bar{H}\left(g_{2}(y), y\right)-K \rho>0,
$$

and

$$
\psi\left(g_{2}(y), \alpha y\right) \varphi\left(g_{1}(y), \alpha y\right)-\psi\left(g_{1}(y), \alpha y\right) \varphi\left(g_{2}(y), \alpha y\right)>0
$$

therefore, $|\Lambda|\left(g_{1}(y), g_{2}(y), y\right)<0$. On the other hand, if item (b) of Assumption 5.1(iii) holds, i.e. if $f_{y x} \equiv 0$ and $f(\cdot, y)$ is strictly convex for any $y \in \mathbb{R}$, we can argue by contradiction as in Case (b) of the proof of Corollary 5.6. To this end, suppose, for example, that $\bar{H}\left(g_{1}\left(y_{o}\right), y_{o}\right)+K \rho=H\left(g_{1}\left(y_{o}\right), y_{o}\right)+K \rho=0$, for some $y_{o} \in \mathbb{R}$. Denoting $f_{y}(x, y)=q(y)$ it then follows that

$$
-\rho K+q\left(y_{o}\right)=-\alpha V_{x}\left(x, y_{o}\right) \quad \forall x \in\left(g_{1}\left(y_{o}\right), \zeta_{1}\left(y_{o}\right)\right),
$$

by definition of $\zeta_{1}$ (cf. Proposition 5.3); that is, $V$ is an affine function of $x$ in that interval. However, using the latter and (4.13), we also have

$$
\frac{1}{\alpha} \alpha y_{o}\left(\rho K-q\left(y_{o}\right)\right)-\rho V\left(x, y_{o}\right)=-f\left(x, y_{o}\right) \quad \forall x \in\left(g_{1}\left(y_{o}\right), \zeta_{1}\left(y_{o}\right)\right)
$$


and we reach a contradiction since $f$ is strictly convex in $x$ by assumption while $V$ is affine. The same argument also implies that $\bar{H}\left(g_{1}\left(y_{o}\right), y_{o}\right)+K \rho \neq 0$. We have then proved that in any case one has $|\Lambda|\left(g_{1}(y), g_{2}(y), y\right) \neq 0$ under Assumption 5.1-(iii).

We can therefore invert the matrix $\Lambda$ appearing in (6.19) and obtain that for a.e. $y \in \mathbb{R}$

$$
\left\{\begin{array}{l}
g_{1}^{\prime}(y)=\frac{1}{|\Lambda|\left(g_{1}(y), g_{2}(y), y\right)}\left[\frac{\partial \Phi_{1}}{\partial x_{2}} \frac{\partial \Phi_{2}}{\partial y}-\frac{\partial \Phi_{2}}{\partial x_{2}} \frac{\partial \Phi_{1}}{\partial y}\right]\left(g_{1}(y), g_{2}(y), y\right)=: G_{1}\left(g_{1}(y), g_{2}(y), y\right) \\
g_{2}^{\prime}(y)=\frac{1}{|\Lambda|\left(g_{1}(y), g_{2}(y), y\right)}\left[\frac{\partial \Phi_{2}}{\partial x_{1}} \frac{\partial \Phi_{1}}{\partial y}-\frac{\partial \Phi_{1}}{\partial x_{1}} \frac{\partial \Phi_{2}}{\partial y}\right]\left(g_{1}(y), g_{2}(y), y\right)=: G_{2}\left(g_{1}(y), g_{2}(y), y\right)
\end{array}\right.
$$

Observe now that, given the aforementioned regularity of $\frac{\partial \Phi_{i}}{\partial x_{j}}, i, j=1,2$, and of $\frac{\partial \Phi_{i}}{\partial y}, i=1,2$, we have $G_{i} \in C_{\text {loc }}^{0, \text { Lip }}(D ; \mathbb{R})$; hence, $g_{i} \in C_{\text {loc }}^{1, \text { Lip }}(\mathbb{R} ; \mathbb{R})$.

Remark 6.5 Notice that the right-hand sides of (6.21) are indeed functions only of $\left(g_{1}(y), g_{2}(y), y\right)$. To see that, it is enough to feed (6.9) and (6.10), and (6.11) and (6.12) in the right-hand sides of (6.21), upon noticing that for any $i, j=1,2, \frac{\partial \Phi_{i}}{\partial x_{j}}$ depend on $A(y), B(y)$, while, for any $i=1,2, \frac{\partial \Phi_{i}}{\partial y}$ depend on $A^{\prime}(y), B^{\prime}(y)$.

Remark 6.6 In the proof of Proposition 5.6 of [20] (see page 2213 therein; see also Step 4 in the proof of Lemma 7 in [35] and the proof of Proposition 6 in [17]), a system of ODEs for the free boundaries is determined with the aim of proving that the free boundaries belong to $C^{1}$ and are strictly monotone. In our problem, proving strict monotonicity of $g_{1}$ and $g_{2}$ would require to establish a strict sign for $G_{1}$ and $G_{2}$ (cf. (6.21)). However, the interaction between our dynamics - and the consequent dependency of $\psi, \varphi$, and $m_{x}$ on $y$ - makes the partial derivatives $\frac{\partial \Phi_{i}}{\partial y}$ appearing in (6.21) much more complex than the analogous quantities in [20] or [35], and this in turn makes it unclear that $G_{i}<0, i=1,2$ (although expected).

\subsection{A Discussion on Theorem 6.4 and the Optimal Control}

\subsubsection{On Theorem 6.4}

Given the full degeneracy of our setting, the fact that the free boundaries $g_{i}, i=1,2$, belong to the class $C_{\text {loc }}^{1, \text { Lip }}(\mathbb{R} ; \mathbb{R})$ is, to the best of our knowledge, a remarkable result. Indeed, the lack of uniform ellipticity of the diffusion coefficient makes it already difficult to obtain a preliminary (locally) Lipschitz property of $g_{i}$ s by invoking results from PDE theory ( [8] and [39], among others) or techniques as those in [40,41], and [42]. Also the probabilistic approach developed in [18] is not directly applicable since our free boundaries are associated with a Dynkin game rather than to an optimal stopping problem.

It is also worth stressing that Theorem 6.4 not only provides regularity of the free boundaries, but also a system of ODEs. To the best of our knowledge, a similar result appears here for the first time. Clearly, in order to provide a complete characterization 


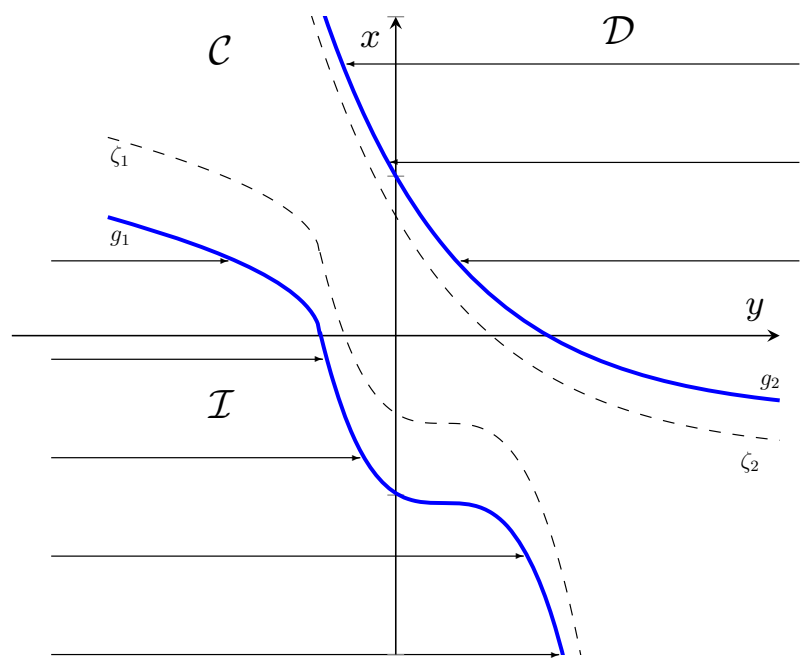

Fig. 1 An illustrative drawing of the free boundaries $g_{1}$ and $g_{2}$ and of the continuation and stopping regions. In the picture, the horizontal lines represent the direction of actions

of $g_{i} \mathrm{~s},(6.15)$ should be complemented by boundary conditions. The determination of those is a non trivial task. As a matter of fact, we have not been able to identify a relevant value of $y$ for which the values of the free boundaries can be determined. The only information available is that the free boundaries diverge for large (in absolute value) levels of $y$; but this is clearly not enough. Even enforcing a finite-fuel constraint like $y \leq Y_{t}^{y, \xi} \leq \bar{y}$ a.s. for any $t \geq 0$ would not help in order to obtain boundary conditions. Indeed, differently to the case with monotone controls (see [32]), here the drift process $Y$ can be pushed back into $(y, \bar{y})$ once any of the boundary points of that interval is reached. Also, it is not clear to us how to obtain some kind of asymptotic growth of the free boundaries in order to restrict the functional class where to look for uniqueness of (6.15).

A possible way to obtain a complete implementable characterization of the free boundaries might be the following. Instead of thinking of $g_{1}$ and $g_{2}$ as functions of $y$, for a fixed parameter $\alpha$, one could look at those as functions of $\alpha$, for any given and fixed $y$. Bearing this in mind, one might try to prove that $\alpha \mapsto g_{i}(\alpha ; y)$ are (at least) locally Lipschitz on $[0, \infty)$, and then follow the approach developed in this section in order to obtain a system of ODEs involving $\partial_{\alpha} g_{i}(\alpha ; y), i=1,2$, rather than $\partial_{y} g_{i}(\alpha ; y)$. Those ODEs would then be complemented by a natural boundary condition since, by continuity, $g_{1}(0+; y)$ and $g_{2}(0+; y)$ would coincide with the free boundaries uniquely determined in Proposition 5.5 of [20]. However, it is not straightforward to prove the aforementioned Lipschitz regularity of $\alpha \mapsto g_{i}(\alpha ; y)$; indeed, a preliminary analysis shows that this is related to that of $\alpha \mapsto V_{x}(x, y ; \alpha)$, and how to prove the latter is not clear to us. The investigation of such an interesting conjecture is therefore left for future research. 


\subsubsection{On the Optimal Control}

Figure 1 provides an illustrative description of the geometry of the state space. The horizontal lines in Fig. 1 represent the directions of the actions induced by the optimal control rule $\xi^{\star}$. This should be such that the jumps of the two-dimensional process $\left(X_{t}^{x, y, \xi^{\star}}, Y_{t}^{y, \xi^{\star}}\right)_{t \geq 0}$ are induced by the optimal control only at initial time, if the initial data $(x, y)$ lie in the interior of $\mathcal{I}$ or $\mathcal{D}$, or at those times at which the process meets jumps of the free boundaries. The size of those interventions should be such that the process is immediately brought to the closest point on $\partial \mathcal{C}$, from where it evolves according to (2.2) and (2.3) and in such a way that it is kept inside the closure of $\mathcal{C}$ in a minimal way. Mathematically, this amounts to construct $\left(X_{t}^{x, y, \xi^{\star}}, Y_{t}^{y, \xi^{\star}}\right)_{t \geq 0}$ as a (degenerate) diffusion that is reflected at $\partial \mathcal{C}$.

The latter is per se an interesting and not trivial problem, whose solution in multidimensional settings strongly hinges on the smoothness of the reflection boundary itself; sufficient conditions can be found in the seminal papers [19] and [33]. Unfortunately, our information on $\partial \mathcal{C}$ do not suffice to apply the results of the aforementioned works since we are not able to exclude horizontal segments of the free boundaries $g_{1}$ and $g_{2}$ (cf. Case (1) and Case (2) in [19]). Indeed, although we can provide explicit formulas for the maps $G_{1}$ and $G_{2}$ appearing in (6.15), their complex expressions makes it hard to show that they are strictly negative (see also Remark 6.6). On the other hand, also the more constructive approach followed in Section 5 of [11] seems not to apply in general to our case, unless we assume (as the authors of [11] do) a linear growth of the free boundaries $b_{i}, i=1,2$, or further requirements on $f$ leading to a weak solution to the reflection problem as in Proposition 7.3 of [21]. We therefore leave for future research the general study of the intricate and intriguing problem of constructing the optimal control.

Acknowledgements Financial support by the German Research Foundation (DFG) through the Collaborative Research Centre 1283 is gratefully acknowledged by the authors.

Open Access This article is licensed under a Creative Commons Attribution 4.0 International License, which permits use, sharing, adaptation, distribution and reproduction in any medium or format, as long as you give appropriate credit to the original author(s) and the source, provide a link to the Creative Commons licence, and indicate if changes were made. The images or other third party material in this article are included in the article's Creative Commons licence, unless indicated otherwise in a credit line to the material. If material is not included in the article's Creative Commons licence and your intended use is not permitted by statutory regulation or exceeds the permitted use, you will need to obtain permission directly from the copyright holder. To view a copy of this licence, visit http://creativecommons.org/licenses/by/4.0/.

\section{References}

1. Ata, B., Harrison, J.M., Shepp, L.A.: Drift control of a Brownian processing system. Ann. Appl. Probab. 15, 1145-1160 (2005)

2. Alvarez, L.H.R.: Optimal capital accumulation under price uncertainty and costly reversibility. J. Econ. Dyn. Control 35, 1769-1788 (2011)

3. Beně̌, V.E.: Girsanov functionals and optimal bang-bang laws for final value stochastic control. Stoch. Process. Appl. 2, 127-140 (1973) 
4. Beně̌, V.E., Shepp, L.A., Witsenhausen, H.S.: Some solvable stochastic control problems. Stochastics 4, 38-83 (1980)

5. Boetius, F.: Bounded variation singular stochastic control and Dynkin games. SIAM J. Control Optim. 44, 1289-1321 (2005)

6. Borodin, A.N., Salminen, P.: Handbook of Brownian Motion- Facts and Formulae, 2nd Corrected Springer, Berlin (2014)

7. Caffarelli, L.A., Crandall, M.G., Kocan, M., Šwiech, A.: On viscosity solutions of fully nonlinear equations with measurable ingredients. Commun. Pure Appl. Math. 49(4), 365-398 (1996)

8. Caffarelli, L.A., Salsa, S.: A Geometric Approach to Free Boundary Problems. Graduate Studies in Mathematics, vol. 68. American Mathematical Society, Providence, RI (2005)

9. Cannarsa, P., Sinestrari, C.: Semiconcave Functions, Hamilton-Jacobi Equations, and Optimal Control. Progress in Nonlinear Differential Equations and Their Applications, vol. 58. Birkhäuser, Basel (2014)

10. Chiarolla, M.B., Haussmann, U.G.: Geometric Approach to Monotone Stochastic Control. Ph.D. Thesis, The University of British Columbia (1992)

11. Chiarolla, M.B., Haussmann, U.G.: Controlling inflation: the infinite horizon case. Appl. Math. Optim. 41, 25-50 (2000)

12. Chow, P.-L., Menaldi, J.-L., Robin, M.: Additive control of stochastic linear systems with finite horizon. SIAM J. Control Optim. 23(6), 858-899 (1985)

13. Chow, P.-L., Menaldi, J.-L.: On the Numerical Solution of a Stochastic Optimal Correction Problem. Transactions of the third army conference on applied mathematics and computing, pp. 531-546 (1986)

14. Clarke, F.H.: Optimization and Nonsmooth Analysis. SIAM, Philadelphia, PA (1990)

15. Dayanik, S., Karatzas, I.: On the optimal stopping problem for one-dimensional diffusions. Stoch. Process. Appl. 107, 173-212 (2003)

16. De Angelis, T., Ferrari, G., Moriarty, J.: A non convex singular stochastic control problem and its related optimal stopping boundaries. SIAM J. Control Optim. 53(3), 1199-1223 (2015)

17. De Angelis, T., Ferrari, G., Moriarty, J.: A solvable two-dimensional degenerate singular stochastic control problem with non convex costs. Math. Oper. Res. 44(2), 512-531 (2019)

18. De Angelis, T., Stabile, G.: On lipschitz continuous optimal stopping boundaries. SIAM J. Control Optim. 57(1), 402-436 (2019)

19. Dupuis, P., Ishii, H.: SDEs with oblique reflection on nonsmooth domains. Ann. Probab. 21(1), 554-580 (1993)

20. Federico, S., Pham, H.: Characterization of the optimal boundaries in reversible investment problems. SIAM J. Control Optim. 52(4), 2180-2223 (2014)

21. Federico, S., Ferrari, G., Schuhmann, P.: A singular stochastic control problem with interconnected dynamics. SIAM J. Control Optim. 58, 2821-2853 (2020)

22. Fleming, W..H., Soner, H..M.: Controlled Markov Processes and Viscosity Solutions, 2nd edn. Springer, Berlin (2005)

23. Guo, X., Tomecek, P.: Connections between singular control and optimal switching. SIAM J. Control Optim. 47, 421-443 (2008)

24. Guo, X., Tomecek, P.: A class of singular control problems and the smooth fit principle. SIAM J. Control Optim. 47, 3076-3099 (2009)

25. Harrison, J.M., Taksar, M.I.: Instantaneous control of brownian motion. Math. Oper. Res. 8(3), 439-453 (1983)

26. Karatzas, I., Ocone, D.: The resolvent of a degenerate diffusion on the plane, with application to partially observed stochastic control. Ann. Appl. Probab. 2, 629-668 (1992)

27. Karatzas, I., Ocone, D.: The finite-horizon version for a partially observed stochastic control problem of Beneš and Rishel. Stoch. Anal. Appl. 11, 569-605 (1993)

28. Karatzas, I., Ocone, D.: A leavable bounded-velocity stochastic control problem. Stoch. Process. Appl. 99, 31-55 (2002)

29. Karatzas, I.: A class of singular stochastic control problems. Adv. Appl. Prob. 15, 225-254 (1983)

30. Karatzas, I., Shreve, S.E.: Connections between optimal stopping and singular stochastic control I. Monotone follower problems. SIAM J. Control Optim 22(6), 856-877 (1984)

31. Karatzas, I., Wang, H.: Connections between bounded-variation control and Dynkin games. In: J.L. Menaldi, A. Sulem and E. Rofman (eds.) Optimal Control and Partial Differential Equations; Volume in Honor of Professor Alain Bensoussan's 60th Birthday, pp. 353-362. IOS Press, Amsterdam (2005)

32. Koch, T., Vargiolu, T.: Optimal Installation of Solar Panels with Price Impact: a Solvable Singular Stochastic Control Problem. (2019) Preprint on arXiv:1911.04223 
33. Lions, P.L., Sznitman, A.S.: Stochastic Differential Equations with Reflecting Boundary Conditions. Commun. Pur. Appl. Math. 37, 511-537 (1984)

34. Matoglu, M.O., Vate, J.V., Wang, H.: Solving the drift control problem. Stoch. Syst. 5(2), 324-371 (2015)

35. Merhi, A., Zervos, M.: A model for reversible investment capacity expansion. SIAM J. Control Optim. 46(3), 839-876 (2007)

36. Papi, M.: On the domain of the implicit function and application. J. Ineq. Appl. 3, 221-234 (2005)

37. Peskir, G.: A change-of-variable formula with local time on curves. J. Theor. Probab. 18(3), 499-535 (2005)

38. Peskir, G.: Optimal stopping games and nash equilibrium. Theory Probab. Appl. 53, 558-571 (2008)

39. Petrosyan, A., Shahgholian, H., Uraltseva, N.: Regularity of Free Boundaries in Obstacle-type Problems. Graduate Studies in Mathematics, vol. 136. American Mathematical Society, Providence, RI (2012)

40. Soner, H.M., Shreve, S.E.: Regularity of the value function for a two-dimensional singular stochastic control problem. SIAM J. Control Optim. 27(4), 876-907 (1989)

41. Soner, H.M., Shreve, S.E.: A Free Boundary Problem Related to Singular Stochastic Control. Applied Stochastic Analysis (London, 1989) 265-301 (1991)

42. Soner, H.M., Shreve, S.E.: A free boundary problem related to singular stochastic control: the parabolic case. Commun. Part. Differ. Equ. 16(2-3), 373-424 (1991)

43. Sun, M., Menaldi, J.-L.: Monotone control of a damped oscillator under random perturbations. IMA J. Math. Control Inform. 5, 169-186 (1988)

44. Urban, T.L.: Inventory models with inventory-level-dependent demand: a comprehensive review and unifying theory. Eur. J. Oper. Res. 162, 792-804 (2005)

45. Taksar, M.I.: Average optimal singular control and a related stopping problem. Math. Oper. Res. 10(1), 63-81 (1985)

46. Yong, J., Zhou, X.Y.: Stochastic Control-Hamiltonian Systems and HJB Equations. Springer, Berlin (1999)

Publisher's Note Springer Nature remains neutral with regard to jurisdictional claims in published maps and institutional affiliations. 